ESAIM: M2AN 50 (2016) 163-185

DOI: $10.1051 / \mathrm{m} 2 \mathrm{an} / 2015039$
ESAIM: Mathematical Modelling and Numerical Analysis

www.esaim-m2an.org

\title{
A MINIMUM-RESIDUAL MIXED REDUCED BASIS METHOD: EXACT RESIDUAL CERTIFICATION AND SIMULTANEOUS FINITE-ELEMENT REDUCED-BASIS REFINEMENT
}

\author{
MASAYUKI YANO ${ }^{1}$
}

\begin{abstract}
We present a reduced basis method for parametrized partial differential equations certified by a dual-norm bound of the residual computed not in the typical finite-element "truth" space but rather in an infinite-dimensional function space. The bound builds on a finite element method and an associated reduced-basis approximation derived from a minimum-residual mixed formulation. The offline stage combines a spatial mesh adaptation for finite elements and a greedy parameter sampling strategy for reduced bases to yield a reliable online system in an efficient manner; the online stage provides the solution and the associated dual-norm bound of the residual for any parameter value in complexity independent of the finite element resolution. We assess the effectiveness of the approach for a parametrized reaction-diffusion equation and a parametrized advection-diffusion equation with a corner singularity; not only does the residual bound provide reliable certificates for the solutions, the associated mesh adaptivity significantly reduces the offline computational cost for the reduced-basis generation and the greedy parameter sampling ensures quasi-optimal online complexity.
\end{abstract}

Mathematics Subject Classification. 65N15, 65N30, 65N35.

Received August 18, 2014. Revised February 4, 2015.

Published online December 1, 2015.

\section{INTRODUCTION}

The certified reduced basis method has emerged as a promising model-reduction strategy to rapidly and reliably solve parametrized partial differential equations (PDEs) in real-time and many-query scenarios [18]. The key to the reliability of the reduced-basis method is a posteriori error bounds. However, to our knowledge, with the few exceptions to be noted shortly, the existing bounds are with respect to some finite-element "truth," which is assumed to be sufficiently accurate and is assumed to be representative of the infinite-dimensional weak solution of the PDE. The assumption is not rigorously validated and, especially for problems with spatial singularities, may be violated in practice. The lack of reliable a posteriori error bounds for the "truth" with respect to the exact infinite-dimensional weak solution leads to either an unreliable reduced-basis approximation with respect to the exact solution or unnecessarily expensive computation of very rich finite-element snapshots in the offline stage. In this work, we develop a reduced-basis method which builds a residual bound relative to

\footnotetext{
Keywords and phrases. Minimum-residual mixed method, reduced basis method, a posteriori error bounds, offline-online decomposition, adaptivity.

1 Department of Mechanical Engineering, Massachusetts Institute of Technology; 77 Massachusetts Ave, Rm. 3-237, Cambridge, MA 02139, United States. myano@mit.edu
} 
the true infinite-dimensional weak solution; we hence aim to remove the issue of truth within the reduced-basis framework.

The residual bound strategy proposed in this work is based on a minimum-residual mixed formulation $[4,14,15]$ of parametrized elliptic PDEs. Our method provides, in the online stage, uniform (as opposed to asymptotic) bounds for the dual norm of the residual computed relative to the infinite-dimensional function space for any parameter value. We insist that our error bounds conform to the standard reduced-basis philosophy as regard its online computational complexity and generality: (1) the bounds are computed in complexity independent of the underlying mixed finite-element discretization; (2) the bounds are available for any parameter value, not just values which belong to the training set used in the offline stage. The proposed minimum-residual mixed formulation provides an approximation of the primal field as well as the dual field, the latter of which then serves to form a bound of the dual norm of the residual. In addition, like other minimum-residual mixed methods and unlike Galerkin mixed methods, the formulation leads to coercive finite-element and reduced-basis systems, which circumvent stability issues associated with for instance the discrete Ladyzenskaja-Babuška-Brezzi (LBB) condition; the guaranteed stability allows us to construct the finite-element and reduced-basis spaces solely from approximation considerations.

We note that the use of the dual variable for a posteriori error estimates - in particular to invoke the complementary variational principle - has been explored in the past. Some of the earlier works in the finite-element community include Ladevèze and Leguillion [9], Ainsworth and Oden [1], and Sauer-Budge et al. [19]. More recent works in the model-reduction community include the application to the proper generalized decomposition by Ladevèze and Chamoin [10] and our application to the reduced-basis method [23]. In particular, in terms of the objective, our earlier work [23] is similar to the current work in that they both seek a strict offline-online decomposition of the exact bound computation for parametrized PDEs. However, in terms of the formulation, our earlier work [23] differs from the current work in that the earlier formulation is based on the reduced-basis approximation of the dual field that satisfies exactly the so-called dual feasibility condition of the complementary variational principle. The formulation of our earlier work [23] suffers from two deficiencies: first, the exact satisfaction of the dual feasibility condition requires, in the offline stage, a non-standard finite-element approximation and, in the online stage, a potentially large algebraic expansion when the number of affine expansion terms is large; second, the application is limited to coercive PDEs.

In order to overcome the limitations of the bound formulations based on the complementary variational principle, we seek in this work the dual-norm bound of the residual relative to the infinite-dimensional function space. We recall that the dual norm of the residual is well-defined even for non-coercive PDEs and is equivalent to the norm of the error (in the sense of (2.3) in Sect. 2). Steih and Urban [21] have recently considered the problem in the context of the reduced basis method; their method, first, utilizes the adaptive wavelet method to compute the reduced-basis snapshots that meet the user-specified true error tolerance and, second, invokes adaptive residual evaluation in the greedy procedure. We note, however, the adaptive online evaluation of the dual norm of the residual for an arbitrary parameter value requires access to the underlying adaptive wavelet method and hence is not online-efficient; the cost of the online certification depends on the complexity of the underlying wavelet discretization. We reiterate that our goal is to provide an online bound that is (1) computed in the complexity independent of the underlying mixed finite-element discretization, and (2) available for any parameter value, not just values which belong to the offline training set.

The contributions of this work are fivefold. First, we develop a mixed formulation for parametrized elliptic equations whose dual variable naturally yields an upper bound of the dual norm of the residual associated with the primal variable. Second, we introduce an associated mixed finite-element and mixed reduced-basis approximations for the weak statement; the approximation is based on the standard Lagrange and Raviart-Thomas elements, which facilitates the implementation within the existing finite-element libraries. Third, we develop an offline-online computational decomposition of the mixed formulation for parametrized PDEs; we pay particular attention to the offline construction of the least-squares mixed system in the parametrized setting. Fourth, we introduce a spatio-parameter adaptation strategy based on an isotropic- $h$ mesh adaptation in space and a greedy 
sampling in parameter. Fifth, and finally, we demonstrate the effectiveness of the proposed strategy applied to elliptic problems with spatial singularities.

We note an important limitation of the current work. While we provide a dual-norm bound of the residual which is equivalent to the norm of the error, we do not provide a lower bound of the stability constant which is required to quantitatively bound the norm of the error. The computation of a lower bound of the stability constant relative to the infinite-dimensional function space is beyond the scope of this work. We however note that residual bounds or even residual estimates have often been found to be sufficient for the purpose of both spatial mesh adaptation for finite element methods [1] and greedy parameter sampling for reduced basis methods $[6,22]$.

This paper is organized as follows. In Section 2, we devise a residual-bound strategy based on the approximation of the primal and dual fields and introduce the associated minimum-residual mixed finite-element and reduced-basis methods. In Section 3, we develop an offline-online computational strategy for the minimumresidual mixed reduced-basis method. In Section 4, we develop a spatial mesh adaptation strategy and a greedy parameter sampling strategy. In Section 5, we apply the method to a reaction-diffusion equation and an advection-diffusion equation.

\section{BOUND CONSTRUCTION}

\subsection{Problem description}

We first introduce a $d$-dimensional bounded domain $\Omega \subset \mathbb{R}^{d}$ with a Lipschitz boundary $\partial \Omega$ such that $\bar{\Gamma}_{N} \cup \bar{\Gamma}_{D}=\overline{\partial \Omega}$ and $\Gamma_{D}$ is nonempty. We also introduce a $P$-dimensional bounded parameter domain $\mathcal{D} \subset \mathbb{R}^{P}$. We then consider parametrized boundary problems of the following form: given a parameter $\mu \in \mathcal{D}$, find $u(\mu)$ that satisfies

$$
\begin{aligned}
\nabla \cdot A(u(\mu), \nabla u(\mu) ; \mu)+C(u(\mu) ; \mu) & =f(\mu) \quad \text { in } \Omega, \\
A(u(\mu), \nabla u(\mu) ; \mu) \cdot \hat{n} & =g(\mu) \quad \text { on } \Gamma_{N}, \\
u(\mu) & =0 \quad \text { on } \Gamma_{D} ;
\end{aligned}
$$

here $A(w, \nabla w ; \mu)$ is a flux operator that is linear in $(w, \nabla w)^{T}, C(w ; \mu)$ is a reaction operator that is linear in $w$, $f(\mu)$ is a source function, $g(\mu)$ is a Neumann boundary function, and $\hat{n}$ is the outward-pointing normal vector. We will shortly state more precise conditions on the flux, reaction, and source operators.

In order to cast the problem in a weak form, we now introduce a Hilbert space

$$
\mathcal{V} \equiv\left\{v \in H^{1}(\Omega)|v|_{\Gamma_{D}}=0\right\}
$$

endowed with an inner product $(w, v)_{\mathcal{V}} \equiv \int_{\Omega} \nabla w \cdot \nabla v \mathrm{~d} x+\int_{\Omega} w v \mathrm{~d} x+\int_{\Gamma_{N}} w v \mathrm{~d} s$ and the induced norm $\|w\|_{\mathcal{V}} \equiv$ $\sqrt{(w, w)_{\mathcal{V}}}$. We then consider the following weak problem: given $\mu \in \mathcal{D}$, find $u(\mu) \in \mathcal{V}$ such that

$$
a(u(\mu), v ; \mu)=\ell(v ; \mu) \quad \forall v \in \mathcal{V},
$$

where

$$
\begin{aligned}
a(w, v ; \mu) & \equiv-\int_{\Omega} \nabla v \cdot A(w, \nabla w ; \mu) \mathrm{d} x+\int_{\Omega} v C(w ; \mu) \mathrm{d} x, \\
\ell(v ; \mu) & \equiv \int_{\Omega} v f(\mu) \mathrm{d} x-\int_{\Gamma_{N}} v g(\mu) \mathrm{d} s \quad \forall v \in \mathcal{V} .
\end{aligned}
$$

We require that $A: \mathcal{V} \times\left(L^{2}(\Omega)\right)^{d} \times \mathcal{D} \rightarrow\left(L^{2}(\Omega)\right)^{d}, C: \mathcal{V} \times \mathcal{D} \rightarrow L^{2}(\Omega), f: \mathcal{D} \rightarrow L^{2}(\Omega)$, and $g: \mathcal{D} \rightarrow L^{2}\left(\Gamma_{N}\right)$; we assume $A(\cdot, \cdot ; \mu), C(\cdot ; \mu), f(\mu)$, and $g(\mu)$ are bounded for all $\mu \in \mathcal{D}$. We also assume, to ensure the problem 
is well-posed, that the bilinear form $a(\cdot, \cdot ; \mu)$ is inf-sup stable and continuous:

$$
\begin{aligned}
& \beta(\mu) \equiv \inf _{w \in \mathcal{V}} \sup _{v \in \mathcal{V}} \frac{a(w, v ; \mu)}{\|w\|_{\mathcal{V}}\|v\|_{\mathcal{V}}}>0 \quad \forall \mu \in \mathcal{D}, \\
& \gamma(\mu) \equiv \sup _{w \in \mathcal{V}} \sup _{v \in \mathcal{V}} \frac{a(w, v ; \mu)}{\|w\|_{\mathcal{V}}\|v\|_{\mathcal{V}}}<\infty \quad \forall \mu \in \mathcal{D} .
\end{aligned}
$$

In addition, we assume that $A, C, f$, and $g$ permit an affine decomposition in the sense that

$$
\begin{aligned}
A(w, \nabla w ; \mu) & =\sum_{q=1}^{Q_{a}} \Theta_{q}^{a}(\mu) A_{q}(w, \nabla w), \\
C(w ; \mu) & =\sum_{q=1}^{Q_{c}} \Theta_{q}^{c}(\mu) C_{q}(w), \\
f(\mu) & =\sum_{q=1}^{Q_{f}} \Theta_{q}^{f}(\mu) f_{q}, \\
g(\mu) & =\sum_{q=1}^{Q_{g}} \Theta_{q}^{g}(\mu) g_{q},
\end{aligned}
$$

for some parameter-independent bounded operators $A_{q}: \mathcal{V} \times\left(L^{2}(\Omega)\right)^{d} \rightarrow\left(L^{2}(\Omega)\right)^{d}, C_{q}: \mathcal{V} \rightarrow L^{2}(\Omega), f_{q} \in$ $L^{2}(\Omega)$, and $g_{q} \in L^{2}\left(\Gamma_{N}\right)$ and parameter-dependent functions $\Theta_{q}^{a}: \mathcal{D} \rightarrow \mathbb{R}, \Theta_{q}^{c}: \mathcal{D} \rightarrow \mathbb{R}, \Theta_{q}^{f}: \mathcal{D} \rightarrow \mathbb{R}$, and $\Theta_{q}^{g}: \mathcal{D} \rightarrow \mathbb{R}$. We note that this is the so called "affine decomposition" assumption that is common to many reduced basis methods [18]; for operators that do not provide an affine decomposition, the empirical interpolation method [2] and its extensions - discrete empirical interpolation method [5], empirical operator interpolation [7], and generalized empirical interpolation method [11] - may provide an approximate decomposition.

Remark 2.1. Many elliptic equations conform to the form introduced above. For instance, a diffusion equation with a parametrized diffusion coefficient $\kappa(\mu) \in \mathbb{R}_{>0}-$ results from $A(w, \nabla w ; \mu)=-\kappa(\mu) \nabla w$. An advectionreaction-diffusion equation - with parametrized diffusion coefficient $\kappa(\mu) \in \mathbb{R}_{>0}$, advection coefficient $\beta(\mu) \in \mathbb{R}^{d}$, and reaction coefficient $\gamma(\mu) \in \mathbb{R}$ - results from $A(w, \nabla w ; \mu)=-\kappa(\mu) \nabla w+\beta(\mu) w$ and $C(w ; \mu)=\gamma(\mu) w$. The Helmholtz equation - with the wavenumber as the parameter - results from $A(w, \nabla w ; \mu)=-\nabla w$ and $C(w ; \mu)=-\mu^{2} w$. The form also supports the variants of the problems with parametrized fields instead of the constants.

We now define the residual. For any $w \in \mathcal{V}$, the residual evaluated relative to some $v \in \mathcal{V}$ is given by

$$
\begin{aligned}
r(v ; w ; \mu) & \equiv \ell(v ; \mu)-a(w, v ; \mu) \\
& =\int_{\Omega} v f(\mu) \mathrm{d} x-\int_{\Gamma_{N}} v g(\mu) \mathrm{d} s+\int_{\Omega} \nabla v \cdot A(w, \nabla w ; \mu) \mathrm{d} x-\int_{\Omega} v C(w ; \mu) \mathrm{d} x .
\end{aligned}
$$

We wish to construct an upper bound of the dual norm of the residual

$$
\|r(\cdot ; w ; \mu)\|_{\mathcal{V}^{\prime}} \equiv \sup _{v \in \mathcal{V}} \frac{|r(v ; w ; \mu)|}{\|v\|_{\mathcal{V}}}
$$


where the element $w \in \mathcal{V}$ arises from a reduced-basis approximation. We note that the residual is related to the error $\|u(\mu)-w\|_{\mathcal{V}}$, for any $w \in \mathcal{V}$, by

$$
\|u(\mu)-w\|_{\mathcal{V}} \leq \frac{1}{\beta(\mu)}\|r(\cdot ; w ; \mu)\|_{\mathcal{V}^{\prime}} \leq \frac{\gamma(\mu)}{\beta(\mu)}\|u(\mu)-w\|_{\mathcal{V}}
$$

where $\beta(\mu)$ is the inf-sup (or stability) constant $(2.1)$ and $\gamma(\mu)$ is the continuity constant (2.2). In this sense, the dual norm of the residual is equivalent to the norm of the error. However, in practice, a lower bound of the inf-sup constant is required to quantitatively bound the norm of the error. As noted in the Introduction, in this work we focus on the computation of an upper bound of the dual norm of the residual and devise a computational strategy that admits an offline-online decomposition; the computation of a lower bound of the inf-sup constant is beyond the scope of this work.

Remark 2.2. The computation of a lower bound of the inf-sup constant (2.1) is difficult, as it requires a lower bound of the eigenvalues associated with a parametrized eigenproblem over an infinite-dimensional function space $\mathcal{V}$. For a problem with very simple domain geometry, boundary conditions, and parametrization, a lower bound of the inf-sup constant can be evaluated analytically; however, we have not been able to devise a computational strategy for more general settings considered here. The computation of a lower bound - which would

enable the construction of an error bound for the solution field (as above) and functional outputs - is an ongoing work.

\subsection{Primal-dual residual bound: theory}

We introduce a Hilbert space

$$
\mathcal{Q} \equiv H(\operatorname{div} ; \Omega) \equiv\left\{q \in\left(L^{2}(\Omega)\right)^{d} \mid \nabla \cdot v \in L^{2}(\Omega)\right\}
$$

endowed with an inner product $(p, q)_{\mathcal{Q}} \equiv(p, q)_{H(\mathrm{div} ; \Omega)} \equiv \int_{\Omega} p \cdot q \mathrm{~d} x+\int_{\Omega}(\nabla \cdot p)(\nabla \cdot q) \mathrm{d} x$ and the induced norm $\|q\|_{\mathcal{Q}} \equiv\|q\|_{H(\operatorname{div} ; \Omega)} \equiv \sqrt{(q, q)_{H(\operatorname{div} ; \Omega)}}$. We are now ready to state the key proposition of this work.

Proposition 2.3. For any $\mu \in \mathcal{D}$ and $w \in \mathcal{V}$, the dual norm of the residual is bounded by

$$
\|r(\cdot ; w ; \mu)\|_{\mathcal{V}^{\prime}} \leq \sqrt{B(w, q ; \mu)} \quad \forall q \in \mathcal{Q}
$$

where

$$
B(w, q ; \mu) \equiv\|f(\mu)-C(w ; \mu)-\nabla \cdot q\|_{L^{2}(\Omega)}^{2}+\|A(w, \nabla w ; \mu)-q\|_{L^{2}(\Omega)}^{2}+\|g(\mu)-q \cdot \hat{n}\|_{L^{2}\left(\Gamma_{N}\right)}^{2} .
$$

Proof. For notational simplicity, in this proof, we suppress the explicit appearance of the parameter $\mu$ in various forms and operators. We first recall that the dual norm may be expressed in terms of its Riesz representation:

$$
\|R(w)\|_{\mathcal{V}}=\|r(\cdot ; w)\|_{\mathcal{V}^{\prime}} \quad \forall w \in \mathcal{V},
$$

where the Riesz representation $R(w) \in \mathcal{V}$ satisfies

$$
(R(w), v)_{\mathcal{V}}=r(v ; w) \quad \forall v \in \mathcal{V}
$$


We then consider the following inequality: for any $v \in \mathcal{V}$,

$$
\begin{aligned}
( & R(w), v)_{\mathcal{V}} \\
= & \int_{\Omega} \nabla v \cdot \nabla R(w) \mathrm{d} x+\int_{\Omega} v R(w) \mathrm{d} x+\int_{\Gamma_{N}} v R(w) \mathrm{d} s \\
= & \int_{\Omega} v f \mathrm{~d} x-\int_{\Gamma_{N}} v g \mathrm{~d} s+\int_{\Omega} \nabla v \cdot A(w, \nabla w) \mathrm{d} x-\int_{\Omega} v C(w) \mathrm{d} x \\
= & \int_{\Omega} v f \mathrm{~d} x-\int_{\Gamma_{N}} v g \mathrm{~d} s+\int_{\Omega} \nabla v \cdot A(w, \nabla w) \mathrm{d} x-\int_{\Omega} v C(w) \mathrm{d} x \\
& -\int_{\Omega} v \nabla \cdot q \mathrm{~d} x+\int_{\Gamma_{N}} v q \cdot \hat{n} \mathrm{~d} s-\int_{\Omega} \nabla v \cdot q \mathrm{~d} x \\
= & \int_{\Omega} v(f-C(w)-\nabla \cdot q) \mathrm{d} x-\int_{\Gamma_{N}} v(g-q \cdot \hat{n}) \mathrm{d} x+\int_{\Omega} \nabla v \cdot(A(w, \nabla w)-q) \mathrm{d} x \\
\leq & \|v\|_{L^{2}(\Omega)}\|f-C(w)-\nabla \cdot q\|_{L^{2}(\Omega)}+\|v\|_{L^{2}\left(\Gamma_{N}\right)}\|g-q \cdot \hat{n}\|_{L^{2}\left(\Gamma_{N}\right)}+\|\nabla v\|_{L^{2}(\Omega)}\|A(w, \nabla w)-q\|_{L^{2}(\Omega)} \\
\leq & \left(\|v\|_{L^{2}(\Omega)}^{2}+\|v\|_{L^{2}\left(\Gamma_{N}\right)}^{2}+\|\nabla v\|_{L^{2}(\Omega)}^{2}\right)^{1 / 2} \\
& \quad\left(\|f-C(w)-\nabla \cdot q\|_{L^{2}(\Omega)}^{2}+\|g-q \cdot \hat{n}\|_{L^{2}\left(\Gamma_{N}\right)}^{2}+\|A(w, \nabla w)-q\|_{L^{2}(\Omega)}^{2}\right)^{1 / 2} \\
= & \|v\|_{\mathcal{V}}(B(w, q))^{1 / 2} ;
\end{aligned}
$$

here the second equality follows from the definition of the residual, the third equality follows from the Green's theorem, the first inequality follows from the triangle inequality, the second inequality follows from Cauchy-Schwarz, and the last equality follows from the definition of the norm $\|\cdot\|_{\mathcal{V}}$ and the form $B(\cdot, \cdot)$. We finally set $v=R(w)$ and invoke the equivalence $\|R(w)\|_{\mathcal{V}}=\|r(\cdot ; w)\|_{\mathcal{V}^{\prime}}$ to obtain the desired result.

Remark 2.4. We may in principle consider more general source functions - namely $f(\mu) \in H^{-1}(\Omega)$ and $g(\mu) \in H^{-1 / 2}\left(\Gamma_{N}\right)$ - by modifying the first inequality in the proof to

$$
\begin{aligned}
\ldots \leq & \|v\|_{H^{1}(\Omega)}\|f-C(w)-\nabla \cdot q\|_{H^{-1}(\Omega)}+\|v\|_{H^{1 / 2}\left(\Gamma_{N}\right)}\|g-q \cdot \hat{n}\|_{H^{-1 / 2}\left(\Gamma_{N}\right)} \\
& +\|\nabla v\|_{L^{2}(\Omega)}\|A(w, \nabla w)-q\|_{L^{2}(\Omega)} .
\end{aligned}
$$

The generalization is important if we wish to treat, for instance, a Dirac delta source function in one dimension. However, the counterpart of the form $B(\cdot, \cdot ; \cdot)$ associated with this decomposition requires the $H^{-1}(\Omega)$ and $H^{-1 / 2}(\Omega)$ norms, which are not readily computable. We hence refrain from the consideration of these more general source functions in this work.

\subsection{Minimum-residual mixed finite element method}

Our reduced-basis approximation, as in the standard reduced-basis approach [18], builds on the finite-element snapshots computed at selected parameter values. We hence first present the finite-element discretization used in this work. We note that the discretization is a generalization of least-squares mixed finite element methods reviewed by Bochev and Gunzburger [4].

We first introduce a sequence of conforming, non-degenerate triangulations $\left\{\mathcal{T}_{h}\right\}$ of $\Omega$; each triangulation $\mathcal{T}_{h}$ consists of non-overlapping triangular elements $\kappa$. We then introduce conforming finite-element approximation spaces for $\mathcal{V}$ and $\mathcal{Q}$ :

$$
\begin{aligned}
\mathcal{V}^{\mathcal{N}_{\mathcal{V}}} & \equiv\left\{v \in \mathcal{V}|v|_{\kappa} \in \mathbb{P}^{p}, \kappa \in \mathcal{T}_{h}\right\}, \\
\mathcal{Q}^{\mathcal{N}_{\mathcal{Q}}} & \equiv\left\{q \in \mathcal{Q}|q|_{\kappa} \in \mathbb{R}^{p-1} \equiv\left(\mathbb{P}^{p-1}\right)^{d} \oplus x \mathbb{P}^{p-1}, \kappa \in \mathcal{T}_{h}\right\} ;
\end{aligned}
$$

note that the space $\mathcal{Q}^{\mathcal{N}_{\mathcal{Q}}}$ consists of Raviart-Thomas elements [16] of degree $p-1$. The superscripts $\mathcal{N}_{\mathcal{V}}$ and $\mathcal{N}_{\mathcal{Q}}$ signify the number of degrees of freedom associated with the spaces $\mathcal{V}^{\mathcal{N}_{\mathcal{V}}}$ and $\mathcal{Q}^{\mathcal{N}_{\mathcal{Q}}}$, respectively. 
Remark 2.5. While we employ Raviart-Thomas's elements for the dual finite-element space $\mathcal{Q}^{\mathcal{N}_{\mathcal{Q}}}$ in this work, our minimum-residual formulation itself admits any conforming approximation space for the dual space. We may for instance consider simple Lagrange polynomial spaces.

We now consider the following minimum-residual bound approximation problem: given $\mu \in \mathcal{D}$, find $\left(u^{\mathcal{N}}(\mu), p^{\mathcal{N}}(\mu)\right) \in \mathcal{V}^{\mathcal{N}_{\mathcal{V}}} \times \mathcal{Q}^{\mathcal{N}_{\mathcal{Q}}}$ such that

$$
\left(u^{\mathcal{N}}(\mu), p^{\mathcal{N}}(\mu)\right)=\underset{\substack{w \in \mathcal{V}^{\mathcal{N}} \\ q \in \mathcal{Q}^{\mathcal{N}} \mathcal{Q}}}{\arg \inf } B(w, q ; \mu),
$$

where $B(\cdot, \cdot ; \cdot)$ is the bound form (2.4). We may identify the Euler-Lagrange's equation associated with the minimization statement: given $\mu \in \mathcal{D}$, find $\left(u^{\mathcal{N}}(\mu), p^{\mathcal{N}}(\mu)\right) \in \mathcal{V}^{\mathcal{N}_{\mathcal{V}}} \times \mathcal{Q}^{\mathcal{N}_{\mathcal{Q}}}$ such that

$$
G\left(\left(u^{\mathcal{N}}(\mu), p^{\mathcal{N}}(\mu)\right),(v, q) ; \mu\right)=L((v, q) ; \mu) \quad \forall v \in \mathcal{V}^{\mathcal{N}_{\mathcal{V}}}, \forall q \in \mathcal{Q}^{\mathcal{N}_{\mathcal{Q}}},
$$

where

$$
\begin{aligned}
G((w, r),(v, q) ; \mu) \equiv & \int_{\Omega}(C(v ; \mu)+\nabla \cdot q)(C(w ; \mu)+\nabla \cdot r) \mathrm{d} x \\
& +\int_{\Omega}(A(v, \nabla v ; \mu)-q) \cdot(A(w, \nabla w ; \mu)-r) \mathrm{d} x \\
& +\int_{\Gamma_{N}}(q \cdot \hat{n})(r \cdot \hat{n}) \mathrm{d} s, \\
L((v, q) ; \mu) \equiv & \int_{\Omega}(C(v ; \mu)+\nabla \cdot q) f(\mu) \mathrm{d} x+\int_{\Gamma_{N}}(q \cdot \hat{n}) g(\mu) \mathrm{d} s ;
\end{aligned}
$$

we take $\mathcal{N} \equiv \mathcal{N}_{\mathcal{V}}+\mathcal{N}_{\mathcal{Q}}$, which serves as a measure of the complexity of the finite-element approximation.

We have the following proposition regards the coercivity of the bilinear form $G(\cdot, \cdot ; \cdot)$.

Proposition 2.6. The bilinear form $G(\cdot, \cdot ; \mu)$ defined in $(2.7)$ is coercive for any $\mu \in \mathcal{D}$ :

$$
G((v, q),(v, q) ; \mu) \geq \alpha(\mu)\left(\|v\|_{\mathcal{V}}^{2}+\|q\|_{\mathcal{Q}}^{2}\right) \quad \forall v \in \mathcal{V}, \forall q \in \mathcal{Q},
$$

for a coercivity constant

$$
\alpha(\mu) \equiv\left[4+\frac{1}{(\beta(\mu))^{2}}\left(1+2\|C(\cdot ; \mu)\|_{\mathcal{L}\left(\mathcal{V}, L^{2}(\Omega)\right)}^{2}+2\|A(\cdot, \cdot ; \mu)\|_{\mathcal{L}\left(\mathcal{V},\left(L^{2}(\Omega)\right)^{d}\right)}^{2}\right)\right]^{-1}
$$

here $\beta(\mu)$ is the inf-sup constant (2.1).

Proof. The proof is provided in Appendix A.

Because the bilinear form is symmetric, bounded (by inspection), and coercive, the problem (2.6) is well-posed. Note in particular the minimum-residual mixed finite element method is not subject to the LBB condition, as noted earlier for coercive PDEs by Pehlivanov et al. $[14,15]$ and discussed in the review for other classes of PDEs by Bochev and Gunzburger [4].

A direct application of Proposition 2.3 shows that the residual associated with our mixed finite-element approximation $u^{\mathcal{N}}(\mu) \in \mathcal{V}^{\mathcal{N} \mathcal{V}}$ is bounded by, for any $\mu \in \mathcal{D}$,

$$
\left\|r\left(\cdot ; u^{\mathcal{N}}(\mu) ; \mu\right)\right\|_{\mathcal{V}^{\prime}}^{2} \leq B\left(u^{\mathcal{N}}(\mu), p^{\mathcal{N}}(\mu) ; \mu\right)
$$

In addition, because the bound functional coincides with the objective function of the minimum-residual statement, the bound is a non-increasing function of $\mathcal{N}$ in the sense that

$$
B\left(u^{\mathcal{N}^{\prime}}(\mu), p^{\mathcal{N}^{\prime}}(\mu) ; \mu\right) \leq B\left(u^{\mathcal{N}}(\mu), p^{\mathcal{N}}(\mu) ; \mu\right)
$$

for nested finite-element spaces $\mathcal{V}^{\mathcal{N}_{\mathcal{V}}^{\prime}} \supset \mathcal{V}^{\mathcal{N}_{\mathcal{V}}}$ and $\mathcal{Q}^{\mathcal{N}_{\mathcal{Q}}^{\prime}} \supset \mathcal{Q}^{\mathcal{N}_{\mathcal{Q}}}$. 


\subsection{Minimum-residual mixed reduced basis method}

We now introduce our reduced-basis approximation. We first introduce an $N$-dimensional primal approximation space spanned by a basis $\left\{\xi_{n} \in \mathcal{V}^{\mathcal{N}_{\mathcal{V}}}\right\}_{n=1}^{N}$,

$$
\mathcal{V}_{N}=\operatorname{span}\left\{\xi_{n}\right\}_{n=1}^{N} \subset \mathcal{V}^{\mathcal{N}_{\mathcal{V}}}
$$

and an $N$-dimensional dual approximation space spanned by a basis $\left\{\eta_{n} \in \mathcal{Q}^{\mathcal{N}_{\mathcal{Q}}}\right\}_{n=1}^{N}$,

$$
\mathcal{Q}_{N}=\operatorname{span}\left\{\eta_{n}\right\}_{n=1}^{N} \subset \mathcal{Q}^{\mathcal{N}_{\mathcal{Q}}} .
$$

We then identify the reduced-basis counterpart of the minimum-residual finite-element statement (2.5): given $\mu \in \mathcal{D}$, find $\left(u_{N}(\mu), p_{N}(\mu)\right) \in \mathcal{V}_{N} \times \mathcal{Q}_{N}$ such that

$$
\left(u_{N}(\mu), p_{N}(\mu)\right)=\underset{\substack{w \in \mathcal{V}_{N} \\ q \in \mathcal{Q}_{N}}}{\arg \inf } B(w, q ; \mu) .
$$

The associated reduced-basis Euler-Lagrange's equation - the counterpart to the finite-element statement (2.6) - is as follows: given $\mu \in \mathcal{D}$, find $\left(u_{N}(\mu), p_{N}(\mu)\right) \in \mathcal{V}_{N} \times \mathcal{Q}_{N}$ such that

$$
G\left(\left(u_{N}(\mu), p_{N}(\mu)\right),(v, q) ; \mu\right)=L((v, q) ; \mu) \quad \forall v \in \mathcal{V}_{N}, \forall q \in \mathcal{Q}_{N},
$$

where the bilinear form $G(\cdot, \cdot ; \cdot)$ and linear form $L(\cdot ; \cdot)$ are as defined in $(2.7)$. Because the bilinear form is symmetric, bounded, and coercive, the reduced-basis system is well-posed. Note in particular the minimumresidual mixed reduced basis method, as in the finite-element counterpart, is not subject to the LBB condition; this is unlike many Galerkin mixed reduced basis methods, which require a careful choice of the spaces ( $c f$. [17]).

Analogous to the finite-element case, a direct application of Proposition 2.3 shows that the residual associated with our mixed reduced-basis approximation $u_{N}(\mu) \in \mathcal{V}_{N}$ is bounded by

$$
\left\|r\left(\cdot ; u_{N}(\mu) ; \mu\right)\right\|_{\mathcal{V}^{\prime}}^{2} \leq B\left(u_{N}(\mu), p_{N}(\mu) ; \mu\right) .
$$

In addition, because the bound functional coincides with the objective function of the minimum-residual statement, the bound is a non-increasing function of $N$ in the sense that

$$
B\left(u_{N^{\prime}}(\mu), p_{N^{\prime}}(\mu) ; \mu\right) \leq B\left(u_{N}(\mu), p_{N}(\mu) ; \mu\right)
$$

for hierarchical reduced-basis spaces $V_{N^{\prime}} \supset V_{N}$ and $Q_{N^{\prime}} \supset Q_{N}$.

Remark 2.7. Because the bilinear form associated with the minimum-residual statement is coercive, we have great flexibility in choosing the reduced-basis approximation spaces. As one example, we could consider primal and dual approximation spaces of different dimensions. As another example, we could consider a reduced-basis approximation in a primal-dual "coupled" space. Namely, in the above "decoupled" formulation, we consider reduced-basis functions of the form $\left(v=\sum_{n=1}^{N} \mathbf{v}_{n} \xi_{n}, q=\sum_{n=1}^{N} \mathbf{q}_{n} \eta_{n}\right)$ for independent coefficient vectors $\mathbf{v} \in \mathbb{R}^{N}$ and $\mathbf{q} \in \mathbb{R}^{N}$; the dimension of the resulting space is $2 N$. We could instead consider a "coupled" formulation, in which we consider reduced-basis functions of the form $\left(v=\sum_{n=1}^{N} \mathbf{c}_{n} \xi_{n}, q=\sum_{n=1}^{N} \mathbf{c}_{n} \eta_{n}\right)$ for a common coefficient vector $\mathbf{c} \in \mathbb{R}^{N}$; the dimension of this space is $N$. For a given set of primal and dual basis functions computed in the offline stage, the decoupled formulation yields a larger online approximation space and hence a smaller residual bound. However, for a fixed dimension of the online reduced-basis approximation space, the coupled formulation often yield a lower residual bound because the coupled formulation takes advantage of the correlation between the parametric manifolds associated with the primal and dual solutions. 


\section{Computational Considerations}

\subsection{Assumption: polynomial form}

We here develop an efficient computational strategy for a special case: the flux operator $A(\cdot, \cdot ; \mu)$ and reaction operator $C(\cdot ; \mu)$ preserve the piecewise polynomial structure of the approximation space $\mathcal{V}^{\mathcal{N} \mathcal{v}}$; the source terms $f(\mu)$ and $g(\mu)$ are piecewise polynomial consistent with the triangulation $\mathcal{T}_{h}$. More precisely, we first introduce element-wise degree- $k$ polynomial discontinuous approximation spaces

$$
\begin{aligned}
\mathcal{X}^{\mathcal{N}_{\mathcal{X}}} & \equiv\left\{x \in L^{2}(\Omega)|x|_{\kappa} \in \mathbb{P}^{k}, \forall \kappa \in \mathcal{T}_{h}\right\}, \\
\mathcal{Y}^{\mathcal{N}_{\mathcal{Y}}} & \equiv\left\{y \in\left(L^{2}(\Omega)\right)^{d}|y|_{\kappa} \in\left(\mathbb{P}^{k}\right)^{d}, \forall \kappa \in \mathcal{T}_{h}\right\}, \\
\mathcal{Z}^{\mathcal{N}_{\mathcal{Z}}} & \equiv\left\{z \in L^{2}(\Gamma)|z|_{\sigma} \in \mathbb{P}^{k}, \sigma \in \Sigma_{h}\right\}
\end{aligned}
$$

here, $\Sigma_{h}$ is the surface triangulation of $\Gamma_{N}$ that is consistent with the volume triangulation $\mathcal{T}_{h}$ of $\Omega$, and $\mathcal{N}_{\mathcal{X}}$, $\mathcal{N}_{\mathcal{Y}}$, and $\mathcal{N}_{\mathcal{Z}}$ are the dimensions of the finite element spaces $\mathcal{X}^{\mathcal{N}_{\mathcal{X}}}, \mathcal{Y}^{\mathcal{N}_{\mathcal{Y}}}$, and $\mathcal{Z}^{\mathcal{N}_{\mathcal{Z}}}$, respectively. We then require that, for all $\mu \in \mathcal{D}$,

$$
\begin{aligned}
A(w, \nabla w ; \mu) & \in \mathcal{Y}^{\mathcal{N}_{\mathcal{Y}}} \quad \forall w \in \mathcal{V}^{\mathcal{N}_{\mathcal{V}}}, \\
C(w ; \mu) & \in \mathcal{X}^{\mathcal{N}_{\mathcal{X}}} \quad \forall w \in \mathcal{V}^{\mathcal{N}_{\mathcal{V}}}, \\
f(\mu) & \in \mathcal{X}^{\mathcal{N}_{\mathcal{X}}} \\
g(\mu) & \in \mathcal{Z}^{\mathcal{N}_{\mathcal{Z}}} .
\end{aligned}
$$

Note that the polynomial degree $k$ need not be the same as the polynomial degree of the space $\mathcal{V}^{\mathcal{N} \mathcal{V}}, p$; in particular we may consider $k>p$ to treat a larger class of operators. We also note that many flux and reaction operators of practical interest preserve the polynomial structure. We refer to Remark 3.1 at the end of the section for considerations in the presence of non-polynomial data and operators.

\subsection{Discrete operators}

We now introduce discrete operators (i.e. matrices) that will be used in our finite-element and reduced-basis approximations. We first introduce a basis $\left\{\chi_{m}\right\}_{m=1}^{\mathcal{N}_{\mathcal{X}}}$ of $\mathcal{X}^{\mathcal{N} \mathcal{X}}$; we assume that the basis functions of the elementwise discontinuous space $\mathcal{X}^{\mathcal{N}_{\mathcal{X}}}$ - as well as those for $\mathcal{Y}^{\mathcal{N}_{\mathcal{Y}}}$ and $\mathcal{Z}^{\mathcal{N}_{\mathcal{Z}}}$ to be introduced shortly - have compact support. We then define an element-wise block matrix $\mathbf{X} \in \mathbb{R}^{\mathcal{N}_{\mathcal{X}} \times \mathcal{N}_{\mathcal{X}}}$ with entries

$$
\mathbf{X}_{m n} \equiv \int_{\Omega} \chi_{m} \chi_{n} \mathrm{~d} x
$$

note that the element-wise block structure arise thanks to the compact support of the basis functions. We next introduce a basis $\left\{\gamma_{m}\right\}_{m=1}^{\mathcal{N}_{\mathcal{Y}}}$ of $\mathcal{Y}^{\mathcal{N}_{\mathcal{Y}}}$ and define an element-wise block matrix $\mathbf{Y} \in \mathbb{R}^{\mathcal{N}_{\mathcal{Y}} \times \mathcal{N}_{\mathcal{Y}}}$ with entries

$$
\mathbf{Y}_{m n} \equiv \int_{\Omega} \gamma_{m} \cdot \gamma_{n} \mathrm{~d} x
$$

We then introduce a basis $\left\{\zeta_{m}\right\}_{m=1}^{\mathcal{N}_{\mathcal{Z}}}$ of $\mathcal{Z}^{\mathcal{N}_{\mathcal{Z}}}$ and define an element-wise block matrix $\mathbf{Z} \in \mathbb{R}^{\mathcal{N}_{\mathcal{Z}} \times \mathcal{N}_{\mathcal{Z}}}$

$$
\mathbf{Z}_{m n} \equiv \int_{\Gamma_{N}} \zeta_{m} \zeta_{n} \mathrm{~d} s
$$


we note that the matrices $\mathbf{X}, \mathbf{Y}$, and $\mathbf{Z}$ are symmetric positive definite. We now introduce a basis $\left\{\psi_{m}\right\}_{m=1}^{\mathcal{N}_{\mathcal{Q}}}$ of $\mathcal{Q}^{\mathcal{N}_{\mathcal{Q}}}$ and define matrices $\mathbf{R} \in \mathbb{R}^{\mathcal{N}_{\mathcal{Y}} \times \mathcal{N}_{\mathcal{Q}}}, \mathbf{D} \in \mathbb{R}^{\mathcal{N}_{\mathcal{X}} \times \mathcal{N}_{\mathcal{Q}}}$, and $\mathbf{B} \in \mathbb{R}^{\mathcal{N}_{\mathcal{Z}} \times \mathcal{N}_{\mathcal{Q}}}$ with entries

$$
\begin{aligned}
\mathbf{R}_{m n} & \equiv \int_{\Omega} \gamma_{m} \cdot \psi_{n} \mathrm{~d} x, \\
\mathbf{D}_{m n} & \equiv \int_{\Omega} \chi_{m}\left(\nabla \cdot \psi_{n}\right) \mathrm{d} x, \\
\mathbf{B}_{m n} & \equiv \int_{\Gamma_{N}} \zeta_{m} \psi_{n} \cdot \hat{n} \mathrm{~d} s .
\end{aligned}
$$

We finally introduce a basis $\left\{\phi_{m}\right\}_{m=1}^{\mathcal{N}_{\mathcal{V}}}$ of $\mathcal{V}^{\mathcal{N}_{\mathcal{V}}}$ and define matrices $\mathbf{A}(\mu) \in \mathbb{R}^{\mathcal{N}_{\mathcal{Y}} \times \mathcal{N}_{\mathcal{V}}}$ and $\mathbf{C}(\mu) \in \mathbb{R}^{\mathcal{N}_{\mathcal{X}} \times \mathcal{N}_{\mathcal{V}}}$ and vectors $\mathbf{f}(\mu) \in \mathbb{R}^{\mathcal{N}_{\mathcal{X}}}$ and $\mathbf{g}(\mu) \in \mathbb{R}^{\mathcal{N}_{\mathcal{Z}}}$ with entries

$$
\begin{aligned}
\mathbf{A}_{m n}(\mu) & \equiv \int_{\Omega} \gamma_{m} \cdot A\left(\phi_{n}, \nabla \phi_{n} ; \mu\right) \mathrm{d} x=\int_{\Omega} \gamma_{m} \cdot \sum_{q=1}^{Q_{a}} \Theta_{q}^{a}(\mu) A^{q}\left(\phi_{n}, \nabla \phi_{n}\right) \mathrm{d} x=\sum_{q=1}^{Q_{a}} \Theta_{q}^{a}(\mu) \mathbf{A}_{m n}^{q}, \\
\mathbf{C}_{m n}(\mu) & \equiv \int_{\Omega} \chi_{m} C\left(\phi_{n} ; \mu\right) \mathrm{d} x=\int_{\Omega} \chi_{m} \sum_{q=1}^{Q_{c}} \Theta_{q}^{c}(\mu) C^{q}\left(\phi_{n}\right) \mathrm{d} x=\sum_{q=1}^{Q_{c}} \Theta_{q}^{c}(\mu) \mathbf{C}_{m n}^{q}, \\
\mathbf{f}_{m}(\mu) & \equiv \int_{\Omega} \chi_{m} f(\mu) \mathrm{d} x=\int_{\Omega} \chi_{m} \sum_{q=1}^{Q_{f}} \Theta_{q}^{f}(\mu) f^{q} \mathrm{~d} x=\sum_{q=1}^{Q_{f}} \Theta_{q}^{f}(\mu) \mathbf{f}_{m}^{q}, \\
\mathbf{g}_{m}(\mu) & \equiv \int_{\Omega} \zeta_{m} g(\mu) \mathrm{d} x=\int_{\Omega} \zeta_{m} \sum_{q=1}^{Q_{g}} \Theta_{q}^{g}(\mu) g^{q} \mathrm{~d} x=\sum_{q=1}^{Q_{g}} \Theta_{q}^{g}(\mu) \mathbf{g}_{m}^{q},
\end{aligned}
$$

where $\mathbf{A}^{q} \in \mathbb{R}^{\mathcal{N}_{\mathcal{Y}} \times \mathcal{N}_{\mathcal{V}}}, q=1, \ldots, Q_{a}, \mathbf{C}^{q} \in \mathbb{R}^{\mathcal{N}_{\mathcal{X}} \times \mathcal{N}_{\mathcal{V}}}, q=1, \ldots, Q_{c}, \mathbf{f}^{q} \in \mathbb{R}^{\mathcal{N}_{\mathcal{X}}}, q=1, \ldots, Q_{f}$, and $\mathbf{g}^{q} \in \mathbb{R}^{\mathcal{N}_{\mathcal{Z}}}$, $q=1, \ldots, Q_{g}$, are given by

$$
\begin{aligned}
\mathbf{A}_{m n}^{q} & \equiv \int_{\Omega} \gamma_{m} \cdot A^{q}\left(\phi_{n}, \nabla \phi_{n}\right) \mathrm{d} x \\
\mathbf{C}_{m n}^{q} & \equiv \int_{\Omega} \chi_{m} C^{q}\left(\phi_{n}\right) \mathrm{d} x \\
\mathbf{f}_{m}^{q} & \equiv \int_{\Omega} \chi_{m} f^{q} \mathrm{~d} x \\
\mathbf{g}_{m}^{q} & \equiv \int_{\Gamma_{N}} \zeta_{m} g^{q} \mathrm{~d} s
\end{aligned}
$$

note that the construction appeals to the affine decomposition of the operators.

\subsection{Implementation of the minimum-residual mixed finite element method}

We first note that, for piecewise-polynomial preserving $A(\cdot, \cdot ; \mu)$ and $C(\cdot ; \mu)$ and piecewise-polynomial source terms $f(\mu)$ and $g(\mu)$, the residual bound operator may be expressed as, for all $w \in \mathcal{V}^{\mathcal{N}_{\mathcal{V}}}$ and $q \in \mathcal{Q}^{\mathcal{N}_{\mathcal{Q}}}$,

$$
\begin{aligned}
B(w, q ; \mu)= & \|f(\mu)-C(w ; \mu)-\nabla \cdot q\|_{L^{2}(\Omega)}^{2}+\|A(w, \nabla w ; \mu)-q\|_{L^{2}(\Omega)}^{2} \\
& +\|g(\mu)-q \cdot \hat{n}\|_{L^{2}\left(\Gamma_{N}\right)}^{2} \\
= & \left\|\Pi_{\mathcal{X}_{\mathcal{N}}}[f(\mu)-C(w ; \mu)-\nabla \cdot q]\right\|_{L^{2}(\Omega)}^{2}+\left\|\Pi_{\mathcal{Y}_{\mathcal{N}} \mathcal{Y}}[A(w, \nabla w ; \mu)-q]\right\|_{L^{2}(\Omega)}^{2} \\
& +\left\|\Pi_{\mathcal{Z}^{\mathcal{N}_{\mathcal{Z}}}}[g(\mu)-q \cdot \hat{n}]\right\|_{L^{2}\left(\Gamma_{N}\right)}^{2},
\end{aligned}
$$

where $\Pi_{\mathcal{X}^{\mathcal{N}_{\mathcal{X}}}}, \Pi_{\mathcal{Y}^{\mathcal{N}_{\mathcal{Y}}}}$, and $\Pi_{\mathcal{Z}^{\mathcal{N}_{\mathcal{Z}}}}$ are the orthogonal projection operators onto $\mathcal{X}^{\mathcal{N}_{\mathcal{X}}}, \mathcal{Y}^{\mathcal{N}_{\mathcal{Y}}}$, and $\mathcal{Z}^{\mathcal{N}_{\mathcal{Z}}}$, respectively. 
We now focus on the first term of $(3.2),\left\|\Pi_{\mathcal{X}_{\mathcal{X}}}[f(\mu)-C(w ; \mu)-\nabla \cdot q]\right\|_{L^{2}(\Omega)}^{2}$, and derive an algebraic form of the expression. We note that, for any $\varsigma \in L^{2}(\Omega)$,

$$
\begin{aligned}
\left\|\Pi_{\mathcal{X} \mathcal{N}_{\mathcal{X}} \varsigma}\right\|_{L^{2}(\Omega)}^{2} & =\left(\Pi_{\mathcal{X}^{\mathcal{N}_{\mathcal{X}}} \varsigma}, \Pi_{\mathcal{X}^{\mathcal{N}_{\mathcal{X}}}} \varsigma\right)_{L^{2}(\Omega)} \\
& =\left(\chi_{m} \mathbf{X}_{m n}^{-1}\left(\chi_{n}, \varsigma\right)_{L^{2}(\Omega)}, \chi_{m^{\prime}} \mathbf{X}_{m^{\prime} n^{\prime}}^{-1}\left(\chi_{n^{\prime}}, \varsigma\right)_{L^{2}(\Omega)}\right)_{L^{2}(\Omega)} \\
& =\left(\chi_{m}, \chi_{m^{\prime}}\right)_{L^{2}(\Omega)} \mathbf{X}_{m n}^{-1} \mathbf{X}_{m^{\prime} n^{\prime}}^{-1}\left(\chi_{n}, \varsigma\right)_{L^{2}(\Omega)}\left(\chi_{n^{\prime}}, \varsigma\right)_{L^{2}(\Omega)} \\
& =\mathbf{X}_{m m^{\prime}} \mathbf{X}_{m n}^{-1} \mathbf{X}_{m^{\prime} n^{\prime}}^{-1}\left(\chi_{n}, \varsigma\right)_{L^{2}(\Omega)}\left(\chi_{n^{\prime}}, \varsigma\right)_{L^{2}(\Omega)}, \\
& =\mathbf{X}_{n n^{\prime}}^{-1}\left(\chi_{n}, \varsigma\right)_{L^{2}(\Omega)}\left(\chi_{n^{\prime}}, \varsigma\right)_{L^{2}(\Omega)},
\end{aligned}
$$

where the summation on the repeated indices is implied. It follows that, for $\varsigma=f(\mu)-C(w, \mu)-\nabla \cdot q=$ $f(\mu)-C\left(\mathbf{w}_{m} \phi_{m} ; \mu\right)-\nabla \cdot \mathbf{q}_{m} \psi_{m}$

$$
\begin{aligned}
\left\|\Pi_{\mathcal{X}^{\mathcal{N}_{\mathcal{X}}}}[f(\mu)-C(w ; \mu)-\nabla \cdot q]\right\|_{L^{2}(\Omega)}^{2}= & \mathbf{X}_{n n^{\prime}}^{-1}\left(\chi_{n}, f(\mu)-C\left(\phi_{m} ; \mu\right) \mathbf{w}_{m}-\nabla \cdot \psi_{m} \mathbf{q}_{m}\right)_{L^{2}(\Omega)}\left(\chi_{n^{\prime}}, f(\mu)\right. \\
& \left.-C\left(\phi_{m^{\prime}} ; \mu\right) \mathbf{w}_{m^{\prime}}-\nabla \cdot \psi_{m^{\prime}} \mathbf{q}_{m^{\prime}}\right)_{L^{2}(\Omega)} \\
= & {[\mathbf{f}(\mu)-\mathbf{C}(\mu) \mathbf{w}-\mathbf{D q}]^{T} \mathbf{X}^{-1}[\mathbf{f}(\mu)-\mathbf{C}(\mu) \mathbf{w}-\mathbf{D q}] } \\
= & \|\mathbf{f}(\mu)-\mathbf{C}(\mu) \mathbf{w}-\mathbf{D q}\|_{\mathbf{X}^{-1}}^{2}
\end{aligned}
$$

We may derive the algebraic expressions for the second and third terms of (3.2) in a similar manner.

We may hence express the residual bound $B(\cdot, \cdot ; \mu)$ defined in $(2.4)$ using the discrete operators as follows: for any $w=\sum_{m=1}^{\mathcal{N}_{\mathcal{V}}} \mathbf{w}_{m} \phi_{m}$ and $q=\sum_{m=1}^{\mathcal{N}_{\mathcal{Q}}} \mathbf{q}_{m} \psi_{m}$,

$$
\begin{aligned}
& B(w, q ; \mu)=\|\mathbf{f}(\mu)-\mathbf{C}(\mu) \mathbf{w}-\mathbf{D} \mathbf{q}\|_{\mathbf{X}^{-1}}^{2}+\|\mathbf{A}(\mu) \mathbf{w}-\mathbf{R} \mathbf{q}\|_{\mathbf{Y}^{-1}}^{2}+\|\mathbf{g}(\mu)-\mathbf{B q}\|_{\mathbf{Z}^{-1}}^{2}
\end{aligned}
$$

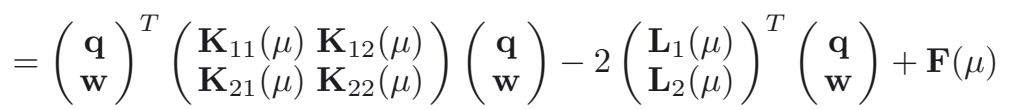

where

$$
\begin{aligned}
\mathbf{K}_{11}(\mu) & \equiv \mathbf{D}^{T} \mathbf{X}^{-1} \mathbf{D}+\mathbf{R}^{T} \mathbf{Y}^{-1} \mathbf{R}+\mathbf{B}^{T} \mathbf{Z}^{-1} \mathbf{B} \\
\mathbf{K}_{12}(\mu) & \equiv \mathbf{K}_{21}^{T}(\mu) \equiv \mathbf{D}^{T} \mathbf{X}^{-1} \mathbf{C}(\mu)-\mathbf{R}^{T} \mathbf{Y}^{-1} \mathbf{A}(\mu) \\
\mathbf{K}_{22}(\mu) & \equiv \mathbf{C}(\mu)^{T} \mathbf{X}^{-1} \mathbf{C}(\mu)+\mathbf{A}(\mu)^{T} \mathbf{Y}^{-1} \mathbf{A}(\mu) \\
\mathbf{L}_{1}(\mu) & \equiv \mathbf{D}^{T} \mathbf{X}^{-1} \mathbf{f}(\mu)+\mathbf{B}^{T} \mathbf{Z}^{-1} \mathbf{g}(\mu) \\
\mathbf{L}_{2}(\mu) & \equiv \mathbf{C}(\mu)^{T} \mathbf{X}^{-1} \mathbf{f}(\mu) \\
\mathbf{F}(\mu) & \equiv \mathbf{f}(\mu)^{T} \mathbf{X}^{-1} \mathbf{f}(\mu)+\mathbf{g}(\mu)^{T} \mathbf{Z}^{-1} \mathbf{g}(\mu)
\end{aligned}
$$

The discrete Euler-Lagrange's equation is the following: find $\left(\mathbf{p}(\mu) \in \mathbb{R}^{\mathcal{N}}, \mathbf{u}(\mu) \in \mathbb{R}^{\mathcal{N}_{\mathcal{Q}}}\right)$ such that

$$
\left(\begin{array}{ll}
\mathbf{K}_{11}(\mu) & \mathbf{K}_{12}(\mu) \\
\mathbf{K}_{21}(\mu) & \mathbf{K}_{22}(\mu)
\end{array}\right)\left(\begin{array}{l}
\mathbf{p}(\mu) \\
\mathbf{u}(\mu)
\end{array}\right)=\left(\begin{array}{l}
\mathbf{L}_{1}(\mu) \\
\mathbf{L}_{2}(\mu)
\end{array}\right)
$$

In practice, we first compute the linear combination of the discrete operators to form $\mathbf{A}(\mu)=\sum_{q=1}^{Q_{a}} \Theta_{q}^{a}(\mu) \mathbf{A}_{q}$, $\mathbf{C}(\mu)=\sum_{q=1}^{Q_{a}} \Theta_{q}^{a}(\mu) \mathbf{C}_{q}, \mathbf{f}(\mu)=\sum_{q=1}^{Q_{a}} \Theta_{q}^{a}(\mu) \mathbf{f}_{q}$, and $\mathbf{g}(\mu)=\sum_{q=1}^{Q_{a}} \Theta_{q}^{a}(\mu) \mathbf{g}_{q}$. We then form the matrices $\mathbf{K}_{i j}(\mu)$, $\mathbf{L}_{i}(\mu)$, and $\mathbf{F}(\mu)$ defined above. We finally solve the linear system. This construction of the discrete system only permitted for the piecewise-polynomial preserving operators - requires computation of $\mathcal{O}\left(\max \left\{Q^{a}, Q^{c}\right\}\right)$ operators and in particular avoids the computation of the $\mathcal{O}\left(\max \left\{Q^{a}, Q^{c}\right\}^{2}\right)$ operators that would arise from a direct computation of the least-squares operators. Note that the latter can be prohibitive for systems that require a large number of affine expansion terms. 
Remark 3.1. For source terms $f(\mu)$ and $g(\mu)$ that are not piecewise polynomial or operators $A(\cdot, \cdot ; \mu)$ and $C(\cdot ; \mu)$ that do not preserve the piecewise polynomial structure, the exact evaluation of the residual bound form (2.4) and the Euler-Lagrange's equation (2.6) becomes difficult. For instance, if $f(\mu)$ is not piecewise polynomial, we inevitably commit quadrature error in the computation of the term $\|f-C(w ; \mu)-\nabla \cdot q\|$. In this case, we may split the term into two and invoke the triangle inequality to obtain $\left\|\Pi_{\mathcal{X}^{\mathcal{N}} \mathcal{X}}(f(\mu)-C(w ; \mu)-\nabla \cdot q)\right\|+$ $\left\|f(\mu)-\Pi_{\mathcal{X} \mathcal{N}_{\mathcal{X}}} f(\mu)\right\|$; if we can construct a bound for the second term arising from the non-polynomial oscillation in $f(\mu)$, then we maintain the rigor in our residual upper bound. We refer to the work of Morin et al. [13] for the treatment of the data oscillation term in a related context of adaptive finite element methods.

\subsection{Implementation of the minimum-residual mixed reduced basis method}

We now consider our reduced-basis implementation. We first note that we may express any $w \in \mathcal{V}_{N} \subset \mathcal{V}^{\mathcal{N}_{\mathcal{V}}}$ as $w=\sum_{n=1}^{N} \sum_{m=1}^{\mathcal{N}_{\mathcal{V}}} \mathbf{V}_{m n} \phi_{m} \boldsymbol{\alpha}_{n}$ for some coefficient vector $\boldsymbol{\alpha} \in \mathbb{R}^{N}$ and a matrix $\mathbf{V} \in \mathbb{R}^{\mathcal{N}_{\mathcal{V}} \times N}$ such that the $n$th reduced basis vector is $\xi_{n}=\sum_{m=1}^{\mathcal{N}_{\mathcal{V}}} \mathbf{V}_{m n} \phi_{m}$. Similarly, we may express any $q \in \mathcal{Q}_{N} \subset \mathcal{Q}^{\mathcal{N}_{\mathcal{Q}}}$ as $q=$ $\sum_{n=1}^{N} \sum_{m=1}^{\mathcal{N}_{\mathcal{Q}}} \mathbf{Q}_{m n} \psi_{m} \boldsymbol{\beta}_{n}$ for some coefficient vector $\boldsymbol{\beta} \in \mathbb{R}^{N}$ and a matrix $\mathbf{Q} \in \mathbb{R}^{\mathcal{N}_{\mathcal{Q}} \times N}$ such that the $n$th reduced basis vector is $\eta_{n}=\sum_{m=1}^{\mathcal{N}_{\mathcal{Q}}} \mathbf{Q}_{m n} \gamma_{n}$. We may thus express the residual bound $B(\cdot, \cdot ; \mu)$ specialized to the reducedbasis spaces using the discrete operators: for any $w=\sum_{n=1}^{N} \sum_{m=1}^{\mathcal{N}_{\mathcal{V}}} \mathbf{V}_{m n} \phi_{m} \boldsymbol{\alpha}_{n}$ and $q=\sum_{n=1}^{N} \sum_{m=1}^{\mathcal{N}_{\mathcal{Q}}} \mathbf{Q}_{m n} \psi_{m} \boldsymbol{\beta}_{n}$,

$$
\begin{aligned}
B(w, q ; \mu)= & \left(\begin{array}{c}
\boldsymbol{\beta} \\
\boldsymbol{\alpha}
\end{array}\right)^{T}\left(\begin{array}{l}
\mathbf{Q}^{T} \mathbf{K}_{11}(\mu) \mathbf{Q} \mathbf{Q}^{T} \mathbf{K}_{12}(\mu) \mathbf{V} \\
\mathbf{V}^{T} \mathbf{K}_{21}(\mu) \mathbf{Q} \mathbf{V}^{T} \mathbf{K}_{22}(\mu) \mathbf{V}
\end{array}\right)\left(\begin{array}{c}
\boldsymbol{\beta} \\
\boldsymbol{\alpha}
\end{array}\right) \\
& -2\left(\begin{array}{c}
\mathbf{Q}^{T} \mathbf{L}_{1}(\mu) \\
\mathbf{V}^{T} \mathbf{L}_{2}(\mu)
\end{array}\right)^{T}\left(\begin{array}{c}
\boldsymbol{\beta} \\
\boldsymbol{\alpha}
\end{array}\right)+\mathbf{F}(\mu) .
\end{aligned}
$$

The discrete reduced-basis Euler-Lagrange's equation is the following: find $\left(\boldsymbol{\alpha}^{*}(\mu) \in \mathbb{R}^{N}, \boldsymbol{\beta}^{*}(\mu) \in \mathbb{R}^{N}\right)$ such that

$$
\left(\begin{array}{l}
\mathbf{Q}^{T} \mathbf{K}_{11}(\mu) \mathbf{Q} \mathbf{Q}^{T} \mathbf{K}_{12}(\mu) \mathbf{V} \\
\mathbf{V}^{T} \mathbf{K}_{21}(\mu) \mathbf{Q} \mathbf{V}^{T} \mathbf{K}_{22}(\mu) \mathbf{V}
\end{array}\right)\left(\begin{array}{c}
\boldsymbol{\beta}^{*}(\mu) \\
\boldsymbol{\alpha}^{*}(\mu)
\end{array}\right)=\left(\begin{array}{c}
\mathbf{Q}^{T} \mathbf{L}_{1}(\mu) \\
\mathbf{V}^{T} \mathbf{L}_{2}(\mu)
\end{array}\right)
$$

Here we provide explicit representations of the reduced-basis matrices using the affine expansion:

$$
\begin{aligned}
\mathbf{Q}^{T} \mathbf{K}_{11} \mathbf{Q} & =\left[\mathbf{Q}^{T} \mathbf{D}^{T} \mathbf{X}^{-1} \mathbf{D} \mathbf{Q}\right]+\left[\mathbf{Q}^{T} \mathbf{R}^{T} \mathbf{Y}^{-1} \mathbf{R} \mathbf{Q}\right]+\left[\mathbf{Q}^{T} \mathbf{B}^{T} \mathbf{Z}^{-1} \mathbf{B} \mathbf{Q}\right] \\
\mathbf{Q}^{T} \mathbf{K}_{12} \mathbf{V} & =\sum_{q=1}^{Q_{c}} \Theta_{q}^{c}(\mu)\left[\mathbf{Q}^{T} \mathbf{D}^{T} \mathbf{X}^{-1} \mathbf{C}^{q} \mathbf{V}\right]-\sum_{q=1}^{Q_{a}} \Theta_{q}^{a}(\mu)\left[\mathbf{Q}^{T} \mathbf{R}^{T} \mathbf{Y}^{-1} \mathbf{A}^{q} \mathbf{V}\right] \\
\mathbf{V}^{T} \mathbf{K}_{22} \mathbf{V} & =\sum_{q^{\prime}, q=1}^{Q_{c}} \Theta_{q^{\prime}}^{c}(\mu) \Theta_{q}^{c}(\mu)\left[\mathbf{V}^{T} \mathbf{C}^{q^{\prime}} \mathbf{X}^{-1} \mathbf{C}^{q} \mathbf{V}\right]+\sum_{q^{\prime}, q=1}^{Q_{a}} \Theta_{q^{\prime}}^{a}(\mu) \Theta_{q}^{a}(\mu)\left[\mathbf{V}^{T} \mathbf{A}^{q^{\prime}} \mathbf{Y}^{-1} \mathbf{A}^{q} \mathbf{V}\right] \\
\mathbf{Q}^{T} \mathbf{L}_{1}(\mu) & \equiv \sum_{q=1}^{Q_{f}} \Theta_{q}^{f}(\mu)\left[\mathbf{Q}^{T} \mathbf{D}^{T} \mathbf{X}^{-1} \mathbf{f}^{q}\right]+\sum_{q=1}^{Q_{g}} \Theta_{q}^{g}(\mu)\left[\mathbf{B}^{T} \mathbf{Z}^{-1} \mathbf{g}^{q}\right], \\
\mathbf{V}^{T} \mathbf{L}_{2}(\mu) & \equiv \sum_{q^{\prime}=1}^{Q_{c}} \sum_{q=1}^{Q_{f}} \Theta_{q}^{c}(\mu) \Theta_{q}^{f}(\mu)\left[\left(\mathbf{C}^{q^{\prime}}\right)^{T} \mathbf{X}^{-1} \mathbf{f}^{q}\right] .
\end{aligned}
$$

The offline-online computational decomposition is clear from the construction. In the offline stage, we compute the finite-element coefficients associated with the reduced basis, $\mathbf{V}$ and $\mathbf{Q}$, and then form the various parameterindependent matrices and their parameter-independent products indicated by brackets in the expression above. In the online stage, we first take appropriate linear combinations of the parameter-independent matrices weighted by parameter-dependent functions to form the reduced-basis Euler-Lagrange's system of size $2 N \times 2 N$; we then solve the reduced-basis system for the coefficient vectors $\boldsymbol{\alpha}^{*}(\mu)$ and $\boldsymbol{\beta}^{*}(\mu)$. 


\section{Spatio-PARAmeter adaptation}

\subsection{Adaptation of the finite element spaces}

Although the ultimate goal is to provide the reduced-basis spaces $\mathcal{V}_{N}$ and $\mathcal{Q}_{N}$, approximations in which meet a user-specified residual tolerance, we first briefly discuss our finite-element adaptation procedure that generates $\mathcal{V}^{\mathcal{N}_{\mathcal{V}}}$ and $\mathcal{Q}^{\mathcal{N}_{\mathcal{Q}}}$ because $\mathcal{V}_{N} \subset \mathcal{V}^{\mathcal{N}_{\mathcal{V}}}$ and $\mathcal{Q}_{N} \subset \mathcal{Q}^{\mathcal{N}_{\mathcal{Q}}}$ by construction. In this work, we consider for simplicity isotropic $h$ adaptivity for a fixed approximation order $p$; however, the residual bound procedure can readily accommodate general anisotropic $h p$ finite-element approximation spaces.

We introduce an elemental residual indicator associated with our finite-element approximations $\left(u^{\mathcal{N}}(\mu), p^{\mathcal{N}}(\mu)\right) \in \mathcal{V}^{\mathcal{N}_{\mathcal{V}}} \times \mathcal{Q}^{\mathcal{N}_{\mathcal{Q}}}:$

$$
\begin{aligned}
\eta_{\kappa} \equiv & \left\|f(\mu)-C\left(u^{\mathcal{N}}(\mu) ; \mu\right)-\nabla \cdot p^{\mathcal{N}}(\mu)\right\|_{L^{2}(\kappa)}^{2}+\left\|A\left(u^{\mathcal{N}}(\mu), \nabla u^{\mathcal{N}}(\mu) ; \mu\right)-p^{\mathcal{N}}(\mu)\right\|_{L^{2}(\kappa)}^{2} \\
& +\left\|g(\mu)-p^{\mathcal{N}}(\mu) \cdot \hat{n}\right\|_{L^{2}\left(\partial \kappa \cap \Gamma_{N}\right)}^{2} \quad \forall \kappa \in \mathcal{T}_{h} ;
\end{aligned}
$$

we observe that

$$
B\left(u^{\mathcal{N}}(\mu), p^{\mathcal{N}}(\mu) ; \mu\right)=\sum_{\kappa \in \mathcal{T}_{h}} \eta_{\kappa} .
$$

Once we identify largest contributors to the residual bound, we employ a fixed-fraction marking strategy to mark elements to refine; in our particular work we refine the top $10 \%$ of the elements with the largest residual indicator. We then refine the marked elements using the newest-vertex bisection algorithm [12]; we recall that the algorithm creates strictly nested meshes such that $\mathcal{V}^{\mathcal{N}_{1}} \subset \mathcal{V}^{\mathcal{N}_{2}} \subset \ldots$ and $\mathcal{Q}^{\mathcal{N}_{1}} \subset \mathcal{Q}^{\mathcal{N}_{2}} \subset \ldots$ for a sequence of adapted meshes. The construction, together with the fact that the solution pair is the minimizer of the residual bound, implies that the residual bound is non-increasing with each mesh refinement.

\subsection{Construction of $\mathcal{V}_{\mathrm{N}}$ and $\mathcal{Q}_{\mathrm{N}}$ : spatio-parameter greedy algorithm}

We construct the reduced-basis spaces $\mathcal{V}_{N}$ and $\mathcal{Q}_{N}$ using a spatio-parameter greedy procedure described in Algorithm 1. We here describe the algorithm in words. We first identify the parameter whose state is leastwell represented in the reduced-basis spaces $\mathcal{V}_{N}$ and $\mathcal{Q}_{N}$ as assessed by the residual bound with respect to the infinite-dimensional function space. We then compute the associated finite-element approximation of the primal and dual fields using the current finite-element discretization, $\mathcal{V}^{\mathcal{N}_{k}}$ and $\mathcal{Q}^{\mathcal{N}_{k}}$. If the residual certificate $B\left(u^{\mathcal{N}_{k}}\left(\tilde{\mu}_{N}\right), p^{\mathcal{N}_{k}}\left(\tilde{\mu}_{N}\right) ; \tilde{\mu}_{N}\right)$ associated with the finite-element solution is greater than the prescribed tolerance, then we adaptively refine the finite-element space until we meet the desired tolerance. We then augment the reduced-basis spaces with these finite-element solutions that meet the required error tolerance.

We make a few remarks. First, if the initial finite-element approximation spaces are sufficiently rich in the sense that $\sup _{\mu \in \Xi_{\text {train }}} \inf _{\substack{w \in \mathcal{V}^{\mathcal{N}_{0}} \\ q \in \mathcal{Q}^{\mathcal{N}_{0}}}} B(w, q ; \mu)<\epsilon_{\text {tol }}^{2}$, then the algorithm works as the standard WeakGreedy algorithm [18]: the finite-element spaces are not enriched as the refinement is not necessary to meet the desired tolerance. Second, whenever the finite-element space is enriched, we re-represent, in terms of the computational implementation, the reduced basis computed on an earlier mesh on the most recent mesh; mathematically, the reduced basis are unaltered since the strictly nested meshes imply that the reduced basis computed on the earlier mesh is exactly representable on all subsequent meshes.

Remark 4.1. In Algorithm 1, we compute finite-element snapshots on successively refined meshes; we then re-represent, in terms of the computational implementation, the reduced basis computed on earlier meshes on the most recent mesh to keep a single finite-element space in which all the snapshots are represented. We could instead compute finite-element snapshots on different meshes specifically adapted for each parameter value; we could then construct a "supermesh" which is a superset of all the finite-element meshes and represent the snapshots on the "supermesh." The latter construction could reduce the cost of finite-element solve, which is 


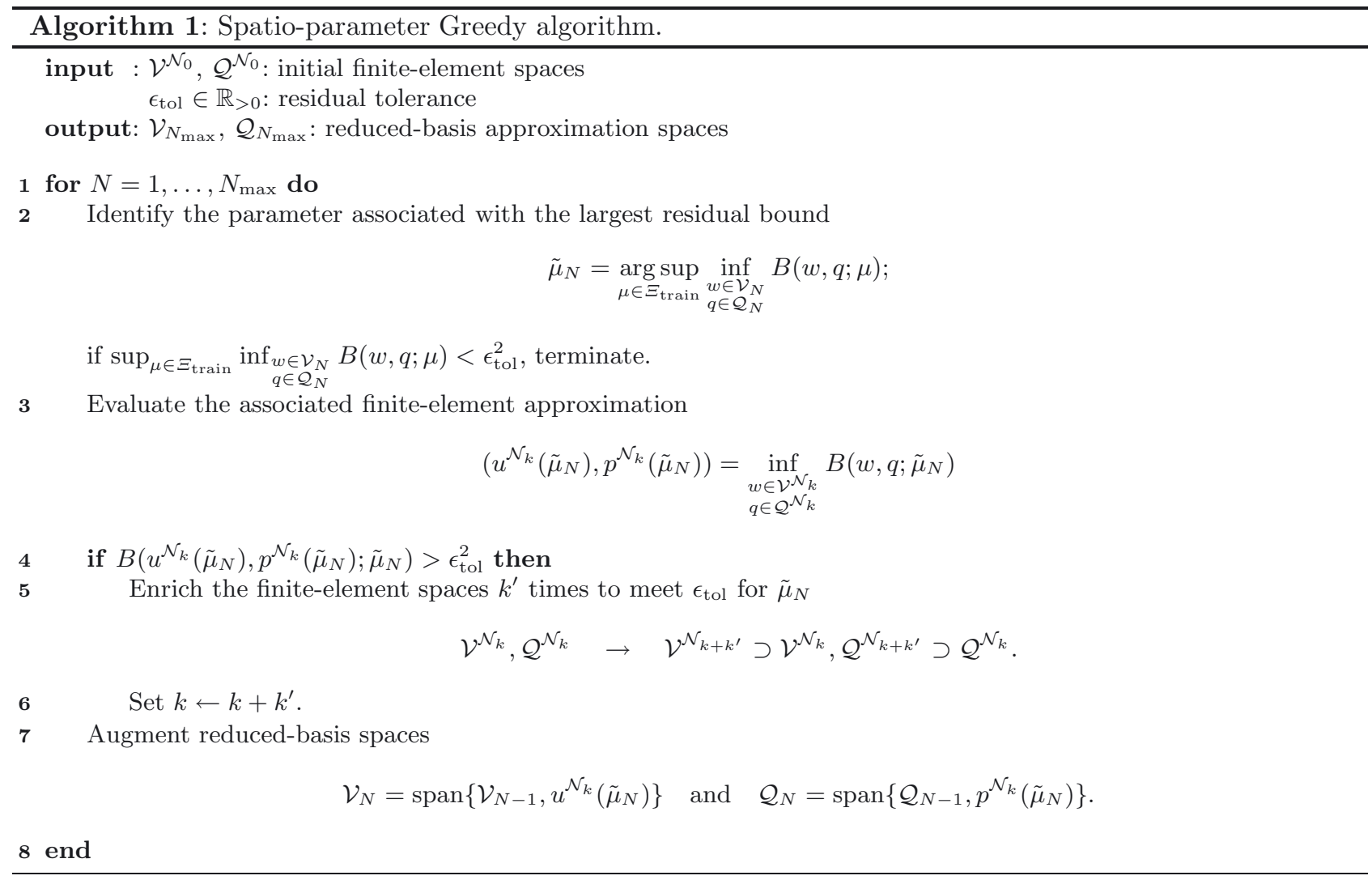

typically superlinear in the number of degrees of freedom. (Note that the "supermesh" is required such that the various inner products required in the computation of (2.8) (or (2.9)) can be performed algebraically as described in Sect. 3).

\section{Numerical Results}

\subsection{Case description}

We consider two problems. The first problem is a parametrized reaction-diffusion problem over a unit square domain, $\Omega \equiv[0,1]^{2}$. The governing equation is given by

$$
\begin{aligned}
-\nabla \cdot(\mu \nabla u)+u & =1 & & \text { in } \Omega, \\
u & =0 & & \text { on } \partial \Omega ;
\end{aligned}
$$

here $\mu \in \mathcal{D} \equiv[0.01,1]$ is the diffusion coefficient. The solution associated with the two extreme values of the parameter are shown in Figure 1; the solution, for the purpose of visualization, is obtained on a uniformly refined mesh with $\mathcal{N} \equiv \mathcal{N}_{\mathcal{V}}+\mathcal{N}_{\mathcal{Q}}=229377$ degrees of freedom. We will use this spatially and parametrically smooth problem to study the convergence behavior of our minimum-residual mixed formulation with spatial refinement and parametric refinement. 


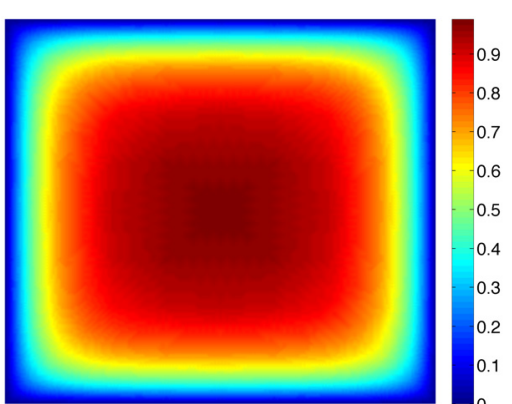

(a) $u(\mu=0.01)$

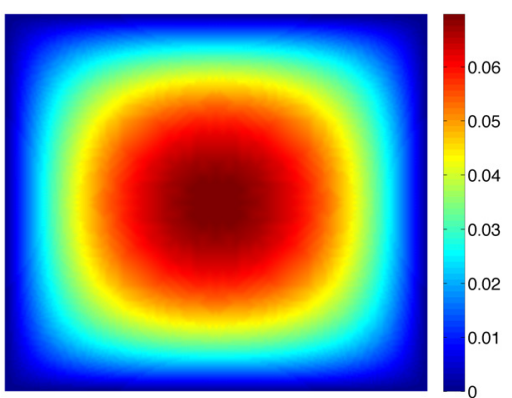

(d) $u(\mu=1)$

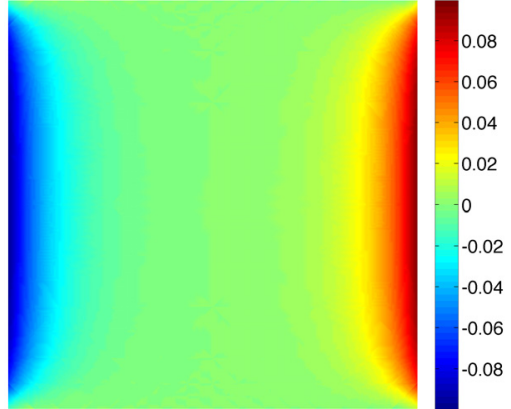

(b) $p_{1}(\mu=0.01)$

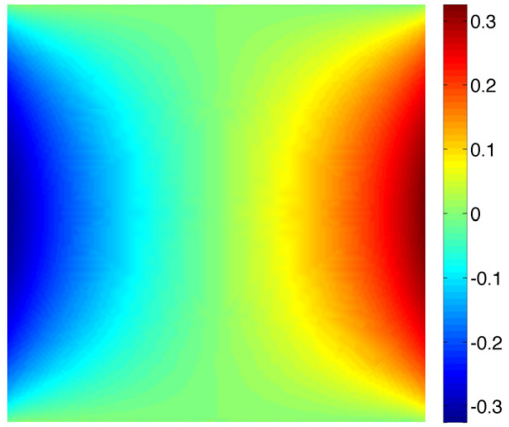

(e) $p_{1}(\mu=1)$

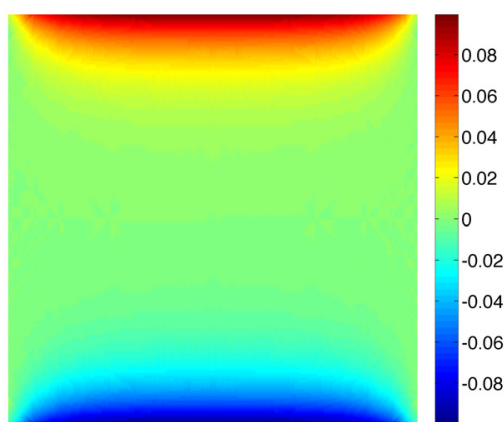

(c) $p_{2}(\mu=0.01)$

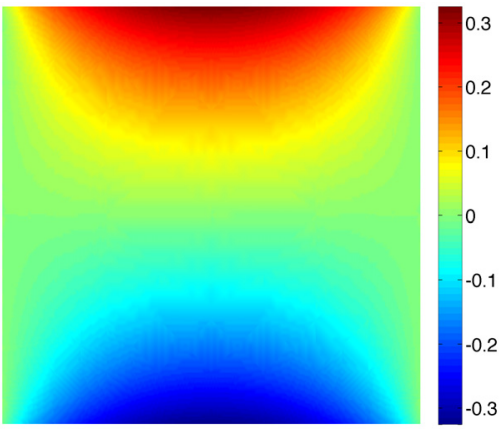

(f) $p_{2}(\mu=1)$

Figure 1. Solution to the reaction-diffusion problem for two different parameter values: $\mu=$ 0.01 and $\mu=1$. Note that $u$ is the primal solution, $p_{1}$ is the first component of the dual solution, and $p_{2}$ is the second component of the dual solution.

The second problem is a parametrized advection-diffusion problem over an $L$-shaped domain, $\Omega \equiv[-1,1]^{2} \backslash$ $[-1,0]^{2}$. The governing equation is given by

$$
\begin{aligned}
-\nabla \cdot(\nabla u)+\nabla \cdot(\beta(\mu) u) & =1 & & \text { in } \Omega, \\
u & =0 & & \text { on } \partial \Omega ;
\end{aligned}
$$

the advection coefficient is given by $\beta(\mu) \equiv(\mu, 0)^{T}$ for the parameter $\mu \in \mathcal{D} \equiv[0,20]$. The solutions associated with the two extreme values of the parameter are shown in Figure 2; the solution, for the purpose of visualization, is obtained on an adaptively refined mesh with $\mathcal{N} \equiv \mathcal{N}_{\mathcal{V}}+\mathcal{N}_{\mathcal{Q}}=9521$ degrees of freedom. Note that, due to the presence of the reentrant corner, the solutions to this problem belong to only $H^{5 / 3-\epsilon}(\Omega)$ for $\epsilon>0$.

We wish to construct a reduced-basis approximation that achieves the residual bound tolerance of $\epsilon_{\text {tol }} \equiv 0.01$ for any $\mu \in \mathcal{D}$. The training parameter set $\Xi_{\text {train }} \subset \mathcal{D}$ for the first problem consists of 201 logarithmically equidistributed points between $[0.01,1]$; the training parameter set for the second problem consists of 201 linearly equidistributed points between $[0,20]$. We will train our online system such that $\epsilon_{\text {tol }}$ is met for all $\mu \in \Xi_{\text {train }}$; we emphasize that the residual bound with respect to the infinite-dimensional function space may be evaluated for any $\mu$ that is not necessarily in $\Xi_{\text {train }}$, but the residual bound tolerance may not be met for $\mu \notin \Xi_{\text {train }}$. We employ $\mathbb{P}^{2} C^{0}$ finite elements for $\mathcal{V}^{\mathcal{N}_{\mathcal{V}}}$ and $\mathbb{R} \mathbb{T}^{1}$ Raviart-Thomas's elements for $\mathcal{Q}^{\mathcal{N}_{\mathcal{Q}}}$.

\subsection{Effectivity of residual bounds}

Before we proceed with convergence analyses, we first assess the effectivity of the residual bound (2.4) for the two cases through numerical experiments. For an finite element approximation $\left(u^{\mathcal{N}}(\mu), p^{\mathcal{N}}(\mu)\right)$, 


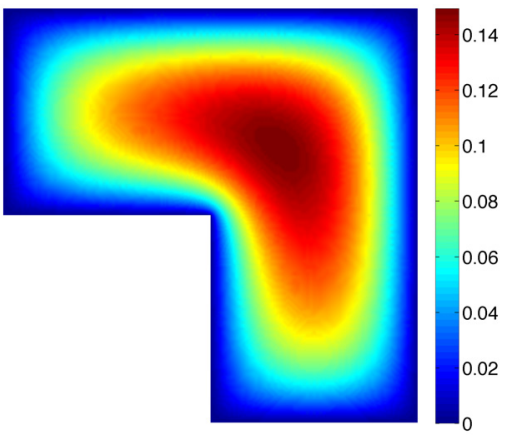

(a) $u(\mu=0)$

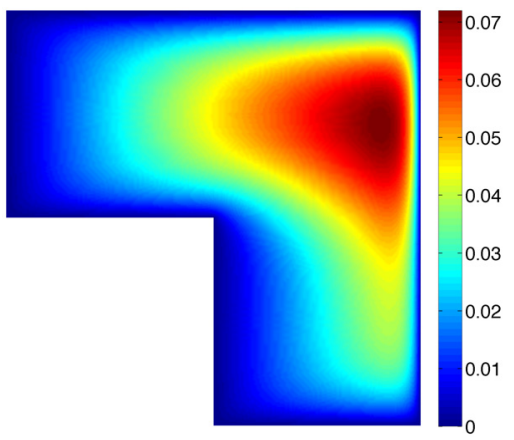

(d) $u(\mu=20)$

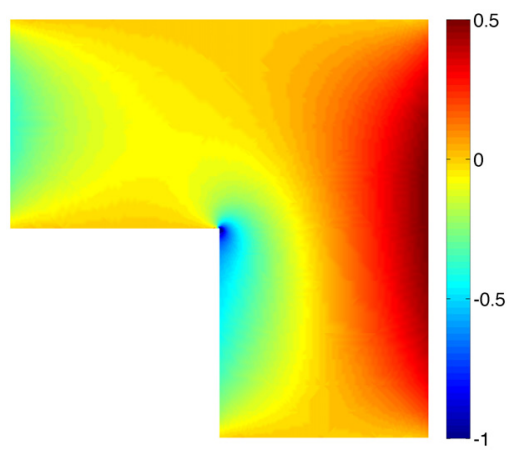

(b) $p_{1}(\mu=0)$

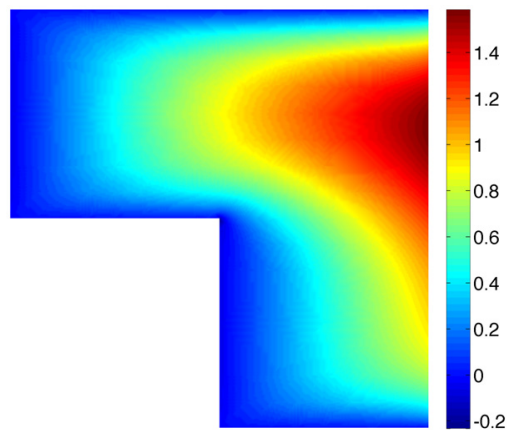

(e) $p_{1}(\mu=20)$

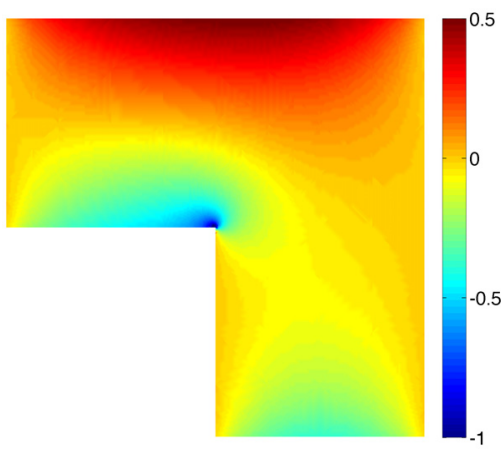

(c) $p_{2}(\mu=0)$

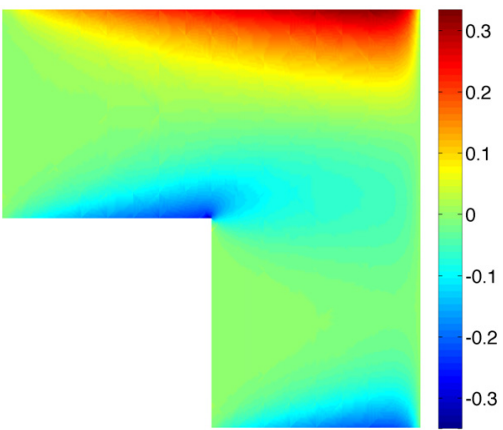

(f) $p_{2}(\mu=20)$

FiguRE 2. Solution to the $L$-shaped advection-diffusion problem for two different parameter values: $\mu=0$ (Poisson) and $\mu=20$. Note that $u$ is the primal solution, $p_{1}$ is the first component of the dual solution, and $p_{2}$ is the second component of the dual solution.

we define the effectivity as the ratio of the residual bound to the dual norm of the residual, $\left[B\left(u^{\mathcal{N}}(\mu), p^{\mathcal{N}}(\mu) ; \mu\right)\right]^{1 / 2} /\left\|r\left(\cdot ; u^{\mathcal{N}}(\mu) ; \mu\right)\right\|_{\mathcal{V}^{\prime}}$. Unfortunately, the effectivity is uncomputable as the dual norm of the residual is uncomputable. We hence instead approximate the dual norm in a fine approximation space $\mathcal{V}^{\hat{N} \mathcal{V}}$; the space $\mathcal{V}^{\hat{N_{V}}}$ is obtained by refining each element of $\mathcal{V}^{\mathcal{N}_{\mathcal{V}}}$ into 64 equal-sized elements. The associated approximation of the effectivity is $\left[B\left(u^{\mathcal{N}}(\mu), p^{\mathcal{N}}(\mu) ; \mu\right)\right]^{1 / 2} /\left\|r\left(\cdot ; u^{\mathcal{N}}(\mu) ; \mu\right)\right\|_{\left(\mathcal{V}_{\mathcal{N}}\right)^{\prime}}$. Note that this approximation is a pessimistic estimate of the exact effectivity, as the approximation of the residual dual norm in the space $\mathcal{V}^{\hat{N} \mathcal{V}} \subset \mathcal{V}$ underestimates the exact residual dual norm with respect to $\mathcal{V}$.

Figure 3a shows the effectivity of the residual bound as a function of the parameter on coarse and finer meshes for the reaction-diffusion's problem. We first confirm that the effectivity is above unity for all parameter values. We also note that the residual bound is quite sharp; the the effectivity is $\mathcal{O}(1)$ for all parameter values. Figure 3b shows the effectivity for the advection-diffusion's problem; we again confirm that the effectivity is above unity and is of $\mathcal{O}(1)$ for all parameter values.

\subsection{Uniform spatio-parameter refinement}

We now assess the behavior of the minimum-residual mixed reduced-basis's method under uniform refinement in spatial and parameter spaces. Specifically, we employ a sequence of (nested) spatially uniform finite-element meshes and a sequence of (non-nested) parametrically equidistributed snapshots.

The convergence of the method applied to the reaction-diffusion problem with the finite-element space dimen$\operatorname{sion} \mathcal{N}$ is shown in Figure 4a; the convergence of the method with the reduced-basis space dimension $N$ is shown 


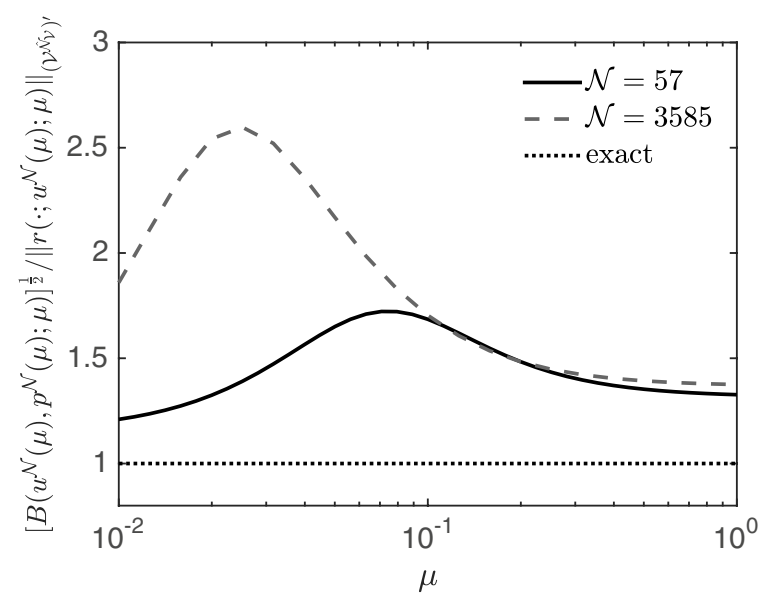

(a) reaction-diffusion

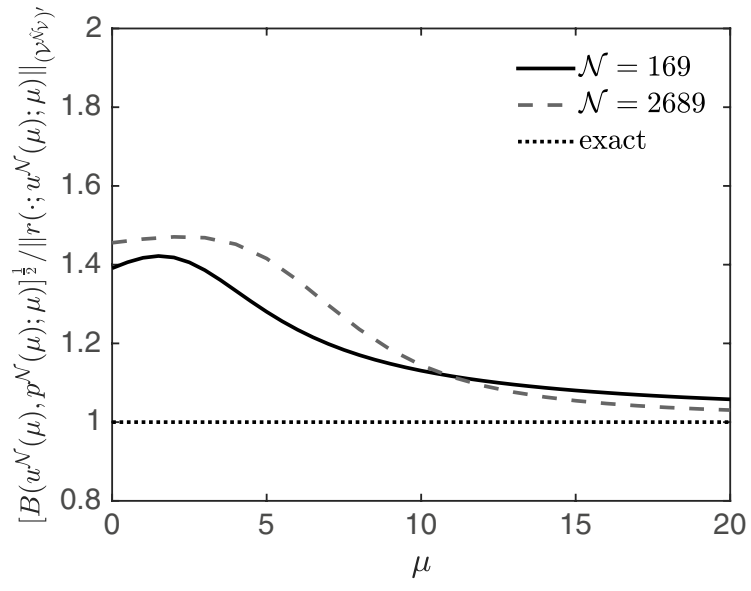

(b) advection-diffusion

Figure 3. The effectivity of the residual bound for the reaction-diffusion and advectiondiffusion problems.

in Figure 4b. In Figure 4a, we observe that, given a sufficient reduced-basis dimension (i.e. $N=4$ ), the residual bound decays at the rate of $\mathcal{N}^{-1}=\mathcal{O}\left(h^{2}\right)$ with finite-element refinement; in other words, we achieve the optimal convergence rate for $\mathbb{P}^{2}-\mathbb{R}^{1}$ mixed elements in the $H^{1}$ norm (which is equivalent to our residual) [14,15]. We also note that, if the reduced-basis space is not sufficiently rich, then the convergence of the residual bound with finite-element refinement stagnates. In Figure 4b, we observe that, given a sufficient finite-element resolution (i.e. $\mathcal{N}=229377$ ), the residual bound decays exponentially with the reduced-basis refinement; this is consistent with the theoretical convergence result anticipated for the smooth parametric manifold [3]. We also note that, if the finite-element space is not sufficiently rich, then the convergence of the residual bound with reduced-basis refinement stagnates. The result signifies the importance of improving both the spatial resolution, through the finite-element enrichment, and the parameter-space resolution, through the reduced-basis enrichment. In the context of the "standard" reduced basis method with the error bounds relative to the finite-element "truth", this result identifies the finite-element fidelity required to rigorously justify the "truth"; such a rigorous validation is in general non-existent and is often overlooked in the "standard" reduced-basis procedure.

We now assess the convergence of the method applied to the advection-diffusion problem with the corner singularity. Figure 5a shows the convergence of the residual certificate with the finite-element space dimension $\mathcal{N}$. We focus the case with a sufficient reduced-basis resolution: $N=6$; we observe that, due to the presence of the singularity, the asymptotic convergence rate is limited to $\mathcal{N}^{-1 / 3}=\mathcal{O}\left(h^{2 / 3}\right)$ despite the use of the $\mathbb{P}^{2}-\mathbb{R}^{1}$ elements. Figure $5 \mathrm{~b}$ shows that we indeed need $\mathcal{N}=172033$ to achieve the desired residual bound of $10^{-2}$; on a smaller finite-element space, we cannot achieve the residual tolerance regardless of the reduced-basis refinement due to the lack of the spatial resolution. We note that the required number of the finite-element degrees of freedom is quite high $-\mathcal{N}=172033$ - due to the presence of the singularity even for this seemingly simple problem. If we wish to obtain a higher-fidelity solution that meets the residual bound of, say, $10^{-3}$, then the asymptotic estimate suggests that we would need $\mathcal{N}=\mathcal{O}\left(10^{8}\right)$ - a prohibitively high computational cost.

\subsection{Adaptive spatio-parameter refinement}

We now invoke the spatio-parameter Greedy algorithm to solve the advection-diffusion problem. We initialize the algorithm with the coarse finite-element mesh shown in Figure 6a that consists of only six elements. The first parameter selected by the algorithm is $\mu=0^{2}$. Since the finite-element error dominates the reduced-basis

\footnotetext{
${ }^{2}$ For $N=0$, the residual is identical for all $\mu \in \mathcal{D}$; the algorithm picks the first occurrence, $\mu=0$, in this case.
} 


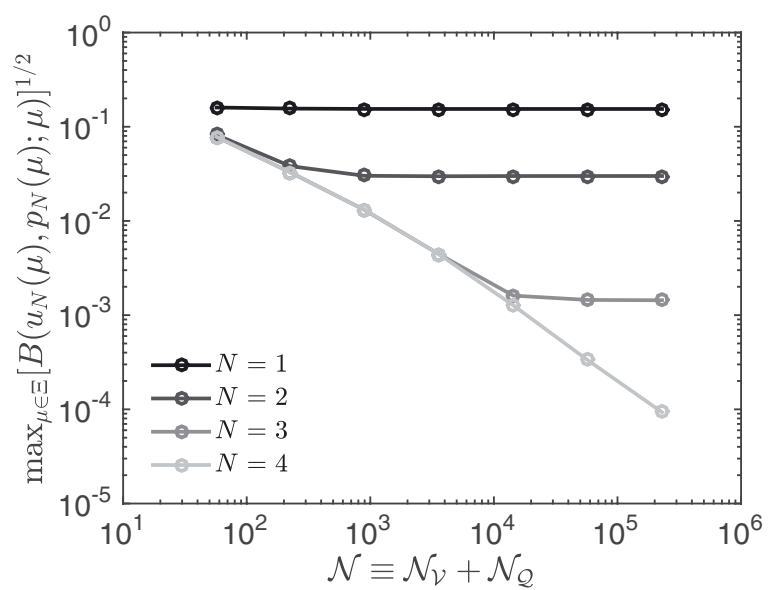

(a) convergence with $\mathcal{N}$

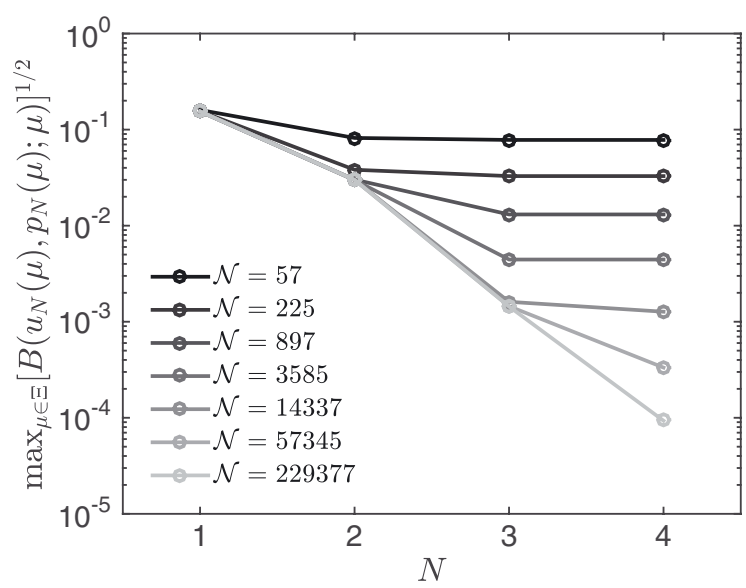

(b) convergence with $N$

FiguRE 4. The reaction-diffusion problem. Convergence of the maximum residual bound over the training set $\Xi_{\text {train }} \subset \mathcal{D}$ with the dimension of (a) the finite-element space and (b) the reduced-basis space.

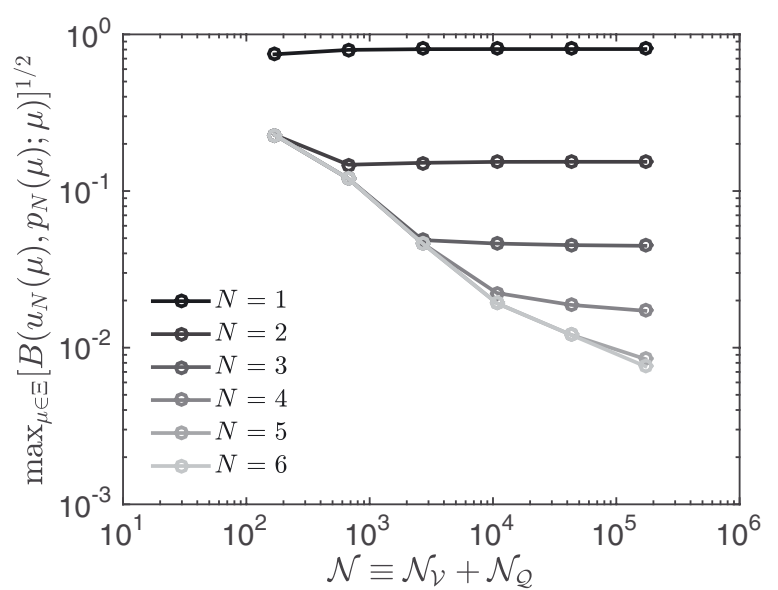

(a) convergence with $\mathcal{N}$

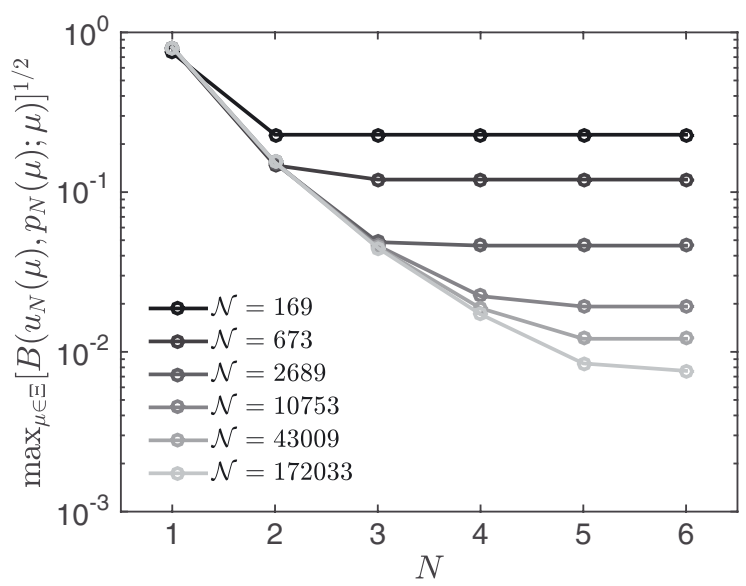

(b) convergence with $N$

FIGURE 5. The advection-diffusion problem. Convergence of the maximum residual bound over the training set $\Xi_{\text {train }} \subset \mathcal{D}$ with the dimension of (a) the finite-element space and (b) the reduced-basis space.

error on this coarse mesh, the algorithm invokes ten steps of adaptive mesh refinement: the number of degrees of freedom increases from 43 to 4495 , and the residual bound for $\mu=0$ reduces from 0.24 to 0.0068 . We emphasize that our formulation provides a bound even on the unreasonable coarse initial mesh, since the bound is uniform and not asymptotic. As shown in Figure $7 \mathrm{a}$, the bound decreases at the rate of $\mathcal{N}^{-1}$, which is the optimal rate for the $\mathbb{P}^{2}-\mathbb{R}^{1}$ discretization despite the presence of the singularity thanks to the adaptive refinement. (for a theoretical analysis, see for instance a monograph by Schwab [20]). The mesh after the ten steps of refinement, $\mathcal{T}_{h}^{k=10}$, is shown in Figure 6b; we observe a localized refinement towards the singular corner.

Upon meeting the residual bound tolerance on $\mathcal{T}_{h}^{k=10}$ for $\mu=0$, the algorithm continues with the greedy sampling of the parameter space. The reduced-basis sampling of the parameter space identifies that the residual 


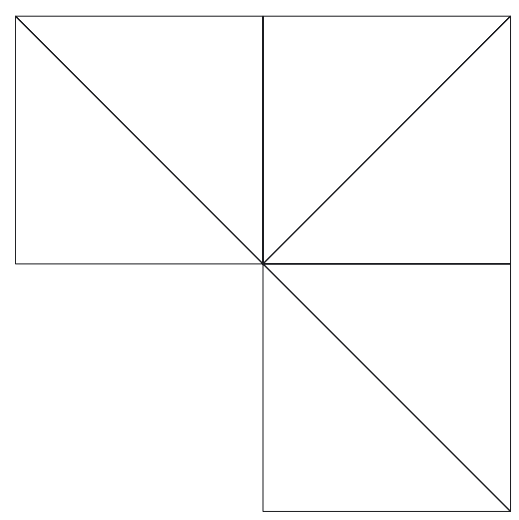

(a) $\mathcal{T}_{h}^{k=0}$

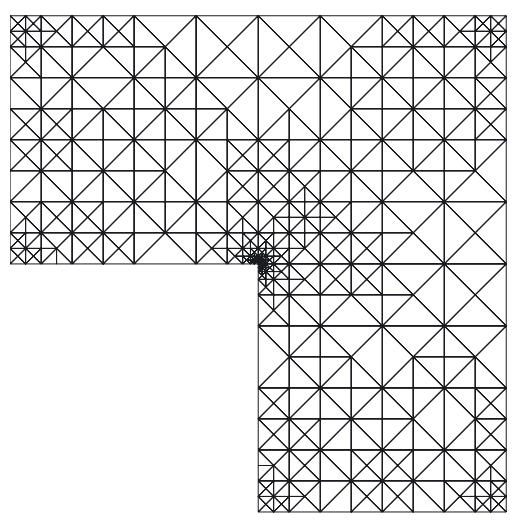

(b) $\mathcal{T}_{h}^{k=10}$

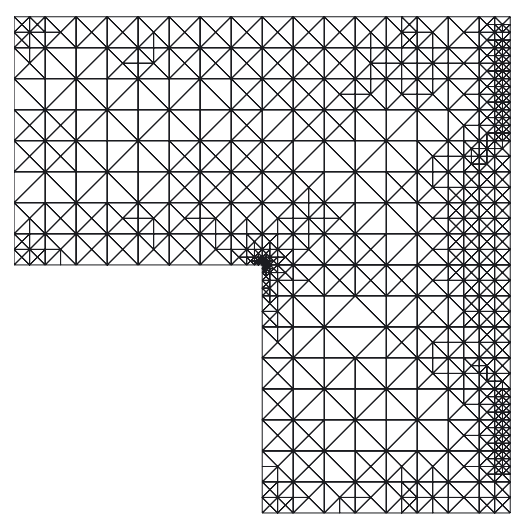

(c) $\mathcal{T}_{h}^{k=12}$

FIgURE 6. finite-element meshes: (a) initial mesh; (b) adapted mesh after refinement at $\mu=0$; (c) adapted mesh after additional refinement at $\mu=20$.

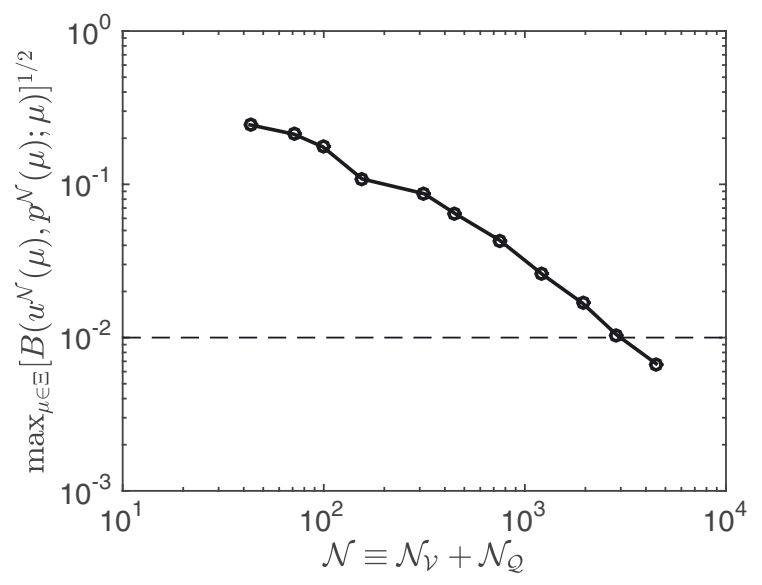

(a) $\mu=0: \mathcal{T}_{h}^{k=0} \rightarrow \mathcal{T}_{h}^{k=10}$

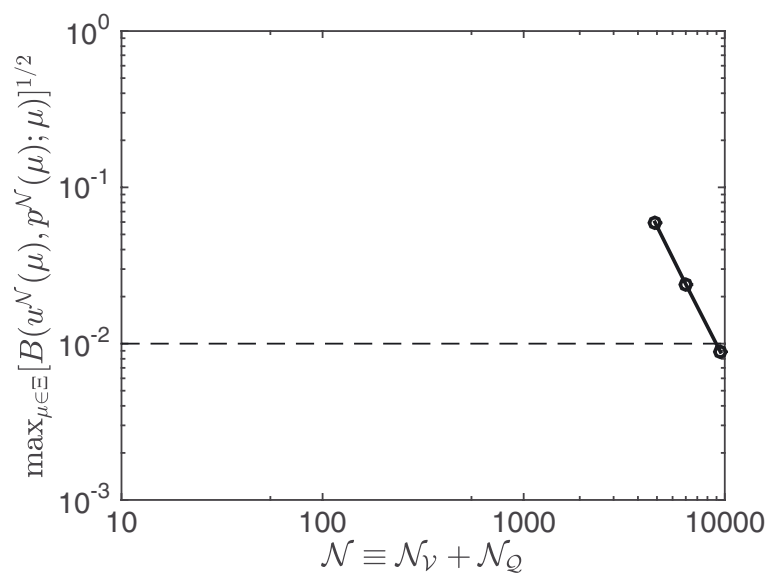

(b) $\mu=20: \mathcal{T}_{h}^{k=10} \rightarrow \mathcal{T}_{h}^{k=12}$

FiguRE 7. Convergence histories for the adaptive finite-element.

bound is maximized for $\mu=20$. The algorithm hence solves for $u^{\mathcal{N}_{10}}(\mu=20)$ and $p^{\mathcal{N}_{10}}(\mu=20)$ with the intention of augmenting $\mathcal{V}_{N=1}=\operatorname{span}\left\{u^{\mathcal{N}_{10}}(\mu=0)\right\}$ and $\mathcal{Q}_{N=1}=\operatorname{span}\left\{p^{\mathcal{N}_{10}}(\mu=0)\right\}$. However, the algorithm identifies that the residual bound associated with $\mu=20$ on $\mathcal{T}_{h}^{k=10}$ is $0.060>\epsilon_{\text {tol }}$. The algorithm hence invokes two additional steps of mesh refinement for $\mu=20$ : the number of degrees of freedom increases from 4495 to 9521 , and the residual bound for $\mu=20$ reduces from 0.060 to 0.0088 . The error decreases rapidly with a relatively small increase in the number of degrees of freedom, as shown in Figure $7 \mathrm{~b}$. The mesh after the two steps of refinement, $\mathcal{T}_{h}^{k=12}$, is shown in Figure 6c; we observe, compared to $\mathcal{T}_{h}^{k=10}$ shown in Figure 6b, additional refinement in the boundary layer.

Upon meeting the residual bound tolerance for $\mu=20$ - and also for $\mu=0$ thanks to the nested finite-element refinement - the algorithm continues with the greedy sampling of the parameter space. This time the algorithm completes the construction of the reduced-basis spaces $\mathcal{V}_{N=5} \subset \mathcal{V}^{\mathcal{N}_{k=12}}$ and $\mathcal{Q}_{N=5} \subset \mathcal{Q}^{\mathcal{N}_{k=12}}$ without requiring 


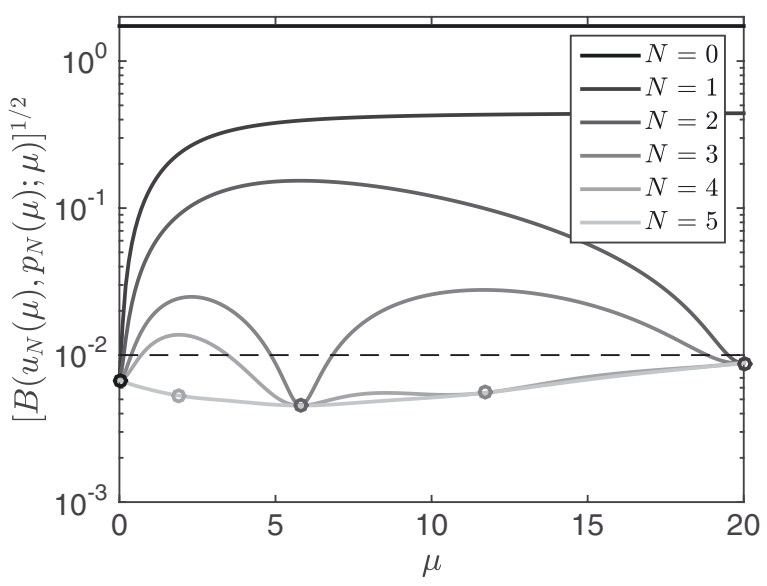

(a) parametric variation

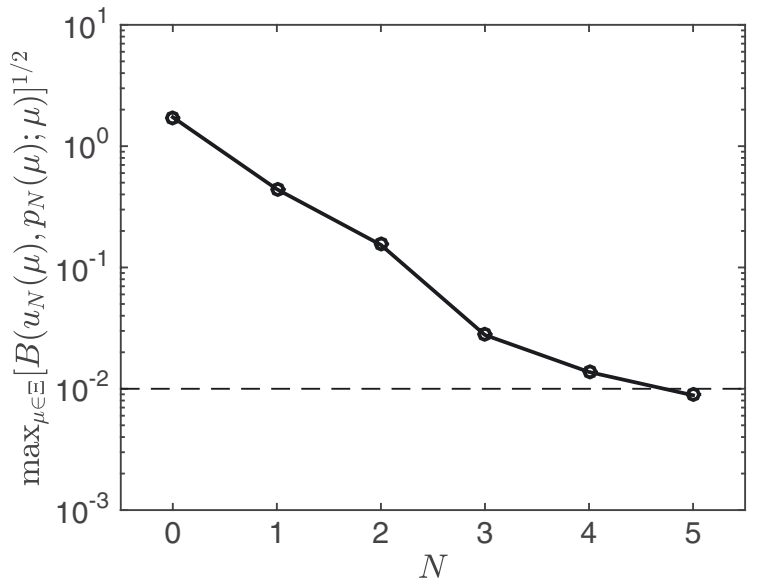

(b) maximum residual

FiguRE 8. Convergence of reduced-basis approximation on $\mathcal{T}_{h}^{k=12}$. The circles (o) in (a) denote the parameters chosen by the greedy algorithm; the shade corresponds to the order in which the parameters are chosen.

additional finite-element enrichment. The greedy convergence over the parameter domain with the dimension of the reduced-basis space, $N$, is shown in Figure 8. With the adaptive finite-element snapshots each of which meets the desired residual tolerance, we observe the exponential convergence with $N$. It is also worth noting the considerable reduction in the number of finite-element degrees of freedom, $\mathcal{N}$, achieved by the adaptive finite-element method compared to the case with uniform meshes.

\section{Summary And Perspectives}

We propose a reduced basis method for parametrized PDEs certified by a bound on the dual norm of the residual computed in an infinite-dimensional functional space. The residual bound builds on a minimum-residual mixed finite element method and the associated mixed reduced basis approximation. We emphasize that our method constructs reduced basis spaces that meet the residual bound tolerance for all training points $\Xi_{\text {train }} \subset \mathcal{D}$, and not just the points about which the snapshots are collected. In addition, in the online stage, we may assess if the reduced-basis approximation meets the required tolerance for any parameter value regardless of whether it belongs to the offline training set. Hence, the greedy algorithm presented here is fundamentally different from a two-step procedure of (1) invoking the standard reduced-basis method that computes the residual relative to the finite-element space - not the infinite-dimensional function space - to identify least-well-represented parameter and (2) invoking an adaptive finite-element method in computing the snapshots; the two-step procedure can in general provide an infinite-dimensional residual bound for only the snapshots.

Because the proposed minimum-residual mixed reduced basis method yields a coercive system and provides a uniform residual bound for any underlying finite-element discretization and any reduced-basis snapshots, we may explore various adaptive strategies. At the finite-element level, we may consider more general $h p$ and anisotropic adaptivity in the physical space [20]. At the reduced-basis level, we may explore $h p$ adaptivity in the parameter space as considered by Eftang et al. [8]; we may also incorporate finer-grain mixing of snapshots computed on different finite-element spaces as considered by Steih and Urban [21]. The design of various spatioparameter adaptive schemes - enabled by the proposed offline-online efficient uniform residual bound - is a key to effectively treat spatially and parametrically complex problems. 


\section{Appendix A. Coercivity of the Bilinear form $G(\cdot, \cdot ; \mu)$}

We prove in this appendix Proposition 2.6, the coercivity of the bilinear form $G(\cdot, \cdot ; \mu)$ : for any $\mu \in \mathcal{D}$,

$$
G((v, q),(v, q) ; \mu) \geq \alpha(\mu)\left(\|v\|_{\mathcal{V}}^{2}+\|q\|_{\mathcal{Q}}^{2}\right) \quad \forall v \in \mathcal{V}, \forall q \in \mathcal{Q}
$$

for

$$
\alpha(\mu)=\left[4+\frac{1}{(\beta(\mu))^{2}}\left(1+2\|C(\cdot ; \mu)\|_{\mathcal{L}\left(\mathcal{V}, L^{2}(\Omega)\right)}^{2}+2\|A(\cdot, \cdot ; \mu)\|_{\mathcal{L}\left(\mathcal{V},\left(L^{2}(\Omega)\right)^{d}\right)}^{2}\right)\right]^{-1}
$$

Proof. For notational simplicity, in this proof, we denote the $L^{2}$ inner product and norm over $\Omega$ without explicit subscripts: that is $(\cdot, \cdot) \equiv(\cdot, \cdot)_{L^{2}(\Omega)}$ and $\|\cdot\| \equiv\|\cdot\|_{L^{2}(\Omega)}$. In addition, we suppress the explicit appearance of the parameter $\mu$ in various forms and operators.

By way of preliminaries, we recall that our bilinear form $a(\cdot, \cdot)$ is inf-sup stable with the inf-sup constant $\beta>0$. We introduce the associated supremizer: $S(w) \in \mathcal{V}$ such that

$$
(S(w), v)_{\mathcal{V}}=a(w, v) \quad \forall v \in \mathcal{V}
$$

note that

$$
\beta \equiv \inf _{w \in \mathcal{V}} \sup _{v \in \mathcal{V}} \frac{a(w, v)}{\|w\|_{\mathcal{V}}\|v\|_{\mathcal{V}}}=\inf _{w \in \mathcal{V}} \frac{\|S(w)\|_{\mathcal{V}}}{\|w\|_{\mathcal{V}}}
$$

We then note that, for any $k \in \mathbb{R}$,

$$
\begin{aligned}
& G((v, q),(v, q))=\|C(v)+\nabla \cdot q\|^{2}+\|A(v, \nabla v)-q\|^{2}+\|q \cdot n\|_{L^{2}\left(\Gamma_{N}\right)}^{2} \\
& =\|C(v)\|^{2}+2(C(v), \nabla \cdot q)+\|\nabla \cdot q\|^{2}+\|A(v, \nabla v)\|^{2}-2(A(v, \nabla v), q)+\|q\|^{2} \\
& +\|q \cdot n\|_{L^{2}\left(\Gamma_{N}\right)}^{2}-2 k(S(v), \nabla \cdot q)-2 k(\nabla S(v), q)+2 k(S(v), q \cdot n)_{L^{2}\left(\Gamma_{N}\right)} \\
& =\|C(v)\|^{2}+2(C(v)-k S(v), \nabla \cdot q)+\|\nabla \cdot q\|^{2} \\
& +\|A(v, \nabla v)\|^{2}-2(A(v, \nabla v)+k \nabla S(v), q)+\|q\|^{2} \\
& +\|q \cdot n\|_{L^{2}\left(\Gamma_{N}\right)}^{2}+2(k S(v), q \cdot n)_{L^{2}\left(\Gamma_{N}\right)} \\
& =\|\nabla \cdot q+(C(v)-k S(v))\|^{2}+\|C(v)\|^{2}-\|C(v)-k S(v)\|^{2} \\
& +\|q-(A(v, \nabla v)+k \nabla S(v))\|^{2}+\|A(v, \nabla v)\|^{2}-\|A(v, \nabla v)+k \nabla S(v)\|^{2} \\
& +\|q \cdot n+k S(v)\|_{L^{2}\left(\Gamma_{N}\right)}^{2}-\|k S(v)\|_{L^{2}\left(\Gamma_{N}\right)}^{2} \\
& =\|\nabla \cdot q+(C(v)-k S(v))\|^{2}+2 k(C(v), S(v))-k^{2}\|S(v)\|^{2} \\
& +\|q-(A(v, \nabla v)+\nabla k S(v))\|^{2}-2 k(A(v, \nabla v), \nabla S(v))-k^{2}\|\nabla S(v)\|^{2} \\
& +\|q \cdot n+k S(v)\|_{L^{2}\left(\Gamma_{N}\right)}^{2}-k^{2}\|S(v)\|_{L^{2}\left(\Gamma_{N}\right)}^{2} \\
& =\|\nabla \cdot q+(C(v)-k S(v))\|^{2}+\|q-(A(v, \nabla v)+k \nabla S(v))\|^{2}+\|q \cdot n+k S(v)\|_{L^{2}\left(\Gamma_{N}\right)}^{2} \\
& +2 k a(v, S(v))-k^{2}\|S(v)\|_{\mathcal{V}}^{2} \\
& \geq 2 k a(v, S(v))-k^{2}\|S(v)\|_{\mathcal{V}}^{2} \\
& =2 k\|S(v)\|_{\mathcal{V}}^{2}-k^{2}\|S(v)\|_{\mathcal{V}}^{2} \\
& =k(2-k)\|S(v)\|_{\mathcal{V}}^{2} \text {. }
\end{aligned}
$$

Some clarifications are in order: the first equality follows from the definition; the second equality follows from the Green's theorem; the fourth equality follows from completing the square; the sixth equality follows from 
$a(w, v)=-(A(w, \nabla w), v)+(C(w), v) \forall w, v \in \mathcal{V}$ and $\|v\|_{\mathcal{V}}^{2}=\|\nabla v\|^{2}+\|v\|^{2}+\|v\|_{L^{2}\left(\Gamma_{N}\right)}^{2} \forall v \in \mathcal{V}$; the second to last equality follows from $a(w, v)=(S(w), v)_{\mathcal{V}} \forall w, v \in \mathcal{V}$. We now set $k=1$ to obtain

$$
G((v, q),(v, q)) \geq\|S(v)\|_{\mathcal{V}}^{2} .
$$

We finally appeal to the definition of the inf-sup constant to obtain

$$
\|v\|_{\mathcal{V}}^{2} \leq \frac{1}{\beta^{2}}\|S(v)\|_{\mathcal{V}}^{2} \leq \frac{1}{\beta^{2}} G((v, q),(v, q))
$$

We next note that

$$
\begin{aligned}
\|\nabla \cdot q\|^{2} & \leq 2\|C(v)+\nabla \cdot q\|^{2}+2\|C(v)\|^{2} \leq 2 G((v, q),(v, q))+2\|C\|_{\mathcal{L}\left(\mathcal{V}, L^{2}(\Omega)\right)}^{2}\|v\|_{\mathcal{V}}^{2} \\
& \leq 2\left(1+\beta^{-2}\|C\|_{\mathcal{L}\left(\mathcal{V}, L^{2}(\Omega)\right)}^{2}\right) G((v, q),(v, q)) \quad \forall v \in \mathcal{V}, \quad \forall q \in \mathcal{Q} .
\end{aligned}
$$

We similarly note that

$$
\begin{aligned}
\|q\|^{2} & \leq 2\|A(v, \nabla v)-q\|^{2}+2\|A(v, \nabla v)\|^{2} \leq 2 G((v, q),(v, q))+2\|A\|_{\mathcal{L}\left(\mathcal{V},\left(L^{2}(\Omega)\right)^{d}\right)}^{2}\|v\|_{\mathcal{V}}^{2} \\
& \leq 2\left(1+\beta^{-2}\|A\|_{\mathcal{L}\left(\mathcal{V},\left(L^{2}(\Omega)\right)^{d}\right)}^{2}\right) G((v, q),(v, q)) \quad \forall v \in \mathcal{V}, \forall q \in \mathcal{Q} .
\end{aligned}
$$

The combination of the last three inequalities yields

$$
\begin{array}{r}
\|v\|_{\mathcal{V}}^{2}+\|q\|_{\mathcal{Q}}^{2} \leq\left[4+\beta^{-2}\left(1+2\|C\|_{\mathcal{L}\left(\mathcal{V}, L^{2}(\Omega)\right)}^{2}+2\|A\|_{\mathcal{L}\left(\mathcal{V},\left(L^{2}(\Omega)\right)^{d}\right)}^{2}\right)\right] \\
\forall v((v, q),(v, q)) \\
\forall v \in \mathcal{V}, \forall q \in \mathcal{Q},
\end{array}
$$

which is the desired inequality.

Acknowledgements. I would like to thank Prof. Anthony Patera of MIT for the many fruitful discussions. This work was supported by OSD/AFOSR/MURI Grant FA9550-09-1-0613 and ONR Grant N00014-11-1-0713.

\section{REFERENCES}

[1] M. Ainsworth and J.T. Oden, A posteriori error estimation in finite element analysis. Comput. Methods Appl. Mech. Engrg. 142 (1997) 1-88.

[2] M. Barrault, Y. Maday, N.C. Nguyen and A.T. Patera, An "empirical interpolation" method: application to efficient reducedbasis discretization of partial differential equations. C. R. Acad. Sci. Paris, Ser. I. 339 (2004) 667-672.

[3] P. Binev, A. Cohen, W. Dahmen, R. DeVore, G. Petrova and P. Wojtaszczyk, Convergence rates for greedy algorithms in reduced basis methods. SIAM J. Math. Anal. 43 (2011) 1457-1472.

[4] P.B. Bochev and M.D. Gunzburger, Finite element methods of least-squares type. SIAM Rev. 40 (1998) $789-837$.

[5] S. Chaturantabut and D.C. Sorensen, Nonlinear model reduction via Discrete Empirical Interpolation. SIAM J. Sci. Comput. 32 (2010) 2737-2764.

[6] W. Dahmen, C. Plesken and G. Welper, Double greedy algorithms: reduced basis methods for transport dominated problems. Math. Model. Numer. Anal. 48 (2013) 623-663.

[7] M. Drohmann, B. Haasdonk and M. Ohlberger, Reduced basis approximation for nonlinear parametrized evolution equations based on empirical operator interpolation. SIAM J. Sci. Comput. 34 (2012) 934-969.

[8] J.L. Eftang, A.T. Patera and E.M. Rønquist, An " $h p$ " certified reduced basis method for parametrized elliptic partial differential equations. SIAM J. Sci. Comput. 32 (2010) 3170-3200.

[9] P. Ladevèze and D. Leguillion, Error estimate procedure in the finite element method and applications. SIAM J. Numer. Anal. 20 (1983) 485-509.

[10] P. Ladevèze and L. Chamoin, On the verification of model reduction methods based on the proper generalized decomposition. Comput. Methods Appl. Mech. Engrg. 200 (2011) 2032-2047. 
[11] Y. Maday and O. Mula, A Generalized Empirical Interpolation Method: Application of Reduced Basis Techniques to Data Assimilation. In Anal. Numer. Partial Differ. Equations, edited by F. Brezzi, P.C. Franzone, U. Gianazza and G. Gilardi. Springer-Verlag (2013) 221-235.

[12] W.F. Mitchell, Adaptive refinement for arbitrary finite-element spaces with hierarchical bases. J. Comput. Appl. Math. 36 (1991) 65-78.

[13] P. Morin, R.H. Nochetto and K.G. Siebert, Convergence of adaptive finite element methods. SIAM Rev. 44 (2002) $631-658$.

[14] A.I. Pehlivanov, G.F. Carey and R.D. Lazarov, Least-squares mixed finite elements for second-order elliptic problems. SIAM J. Numer. Anal. 31 (1994) 1368-1377.

[15] A.I. Pehlivanov, G.F. Carey and P.S. Vassilevski, Least-squares mixed finite element methods for non-selfadjoint elliptic problems: I. Error estimates. Numer. Math. 72 (1996) 501-522.

[16] P.A. Raviart and J.M. Thomas, A Mixed Finite Element Method for 2nd Order Elliptic Problems. In Vol. 606 of Lect. Notes Math. Springer-Verlag (1977) 292-315.

[17] G. Rozza and K. Veroy, On the stability of the reduced basis method for stokes equations in parametrized domains. Comput. Methods Appl. Mech. Engrg. 196 (2007) 1244-1260.

[18] G.Rozza, D.B.P. Huynh and A.T. Patera, Reduced basis approximation and a posteriori error estimation for affinely parametrized elliptic coercive partial differential equations - application to transport and continuum mechanics. Arch. Comput. Methods Eng. 15 (2008) 229-275.

[19] A.M. Sauer-Budge, J. Bonet, A. Huerta and J. Peraire, Computing bounds for linear functionals of exact weak solutions to Poisson's equation. SIAM J. Numer. Anal. 42 (2004) 1610-1630.

[20] C. Schwab, $p$ - and $h p$-Finite Element Methods. Oxford Science Publications, Great Clarendon Street, Oxford, UK (1998).

[21] K. Steih and K. Urban, Reduced basis methods based upon adaptive snapshot computations. Preprint arxiv: 1407.1708v1 (2014).

[22] T. Tonn, K. Urban and S. Volkwein, Comparison of the reduced-basis and POD a posteriori error estimators for an elliptic linear-quadratic optimal control problem. Math. Comput. Model. Dyn. 17 (2011) 355-369.

[23] M. Yano, A reduced basis method with exact-solution certificates for steady symmetric coercive equations. Comput. Methods Appl. Mech. Engrg. 287 (2015) 290-309. 